\title{
TRANSFORMATION GROUPS ON COMPACT SYMMETRIC SPACES
}

\author{
BY \\ TADASHI NAGANO (1)
}

The symmetric spaces constitute the most important class of Riemannian manifolds; some of them have been the standard spaces in various branches of geometry, and many authors threw light upon their deep properties. Still there seems to be no thorough study of general transformation groups $L$ (other than the isometry groups $H$, but containing $G$ ) of compact $\left({ }^{2}\right)$ symmetric spaces $M$, which we call geometric transformation groups of $M$ in this introduction. Its need will be patent if it will reveal interrelations of symmetric spaces, and if $L$ will be the automorphism group of some geometric structure of $M$, more or less closely related with the Riemannian structure of $M$, by which $M$ is geometrically distinguished from the other symmetric spaces.

Let us observe a few examples. Let $M$ be the sphere as a symmetric space. $M$ has the projective [respectively, conformal] structure; it can be thought of as the set of all geodesics [respectively, the function which gives the angles between two tangent vectors at the same points] of $M$. This can be defined, of course, for any Riemannian manifold, but the automorphism group $L$, or the projective [respectively, conformal] transformation group, differs from the isometry group $G$ for the sphere $M$, and by this fact, $M$ is distinguished from all other symmetric spaces (except the real projective space which is locally isometric with $M$ ), as asserted by E. Cartan [Oeuvres complètes, Partie I, Vol. II, Gauthier-Villars, Paris, 1952, p. 659]. (See [7], [8] for the proof.) $M$ is the standard space in the projective [respectively, conformal] differential geometry. And, à la F. Klein, this group $L$ on $M$ gives rise to the (real) projective [respectively, conformal or Moebius] geometry. Next, to observe another example, we select a compact hermitian symmetric space for $M$. The structure is the complex structure connected with the Riemannian metric in a certain way. The automorphism group $L$ is the holomorphic transformation group. $L$ is a complex Lie group whose complex structure essentially determines that of $M$.

Received by the editors February 26, 1964.

(1) The author thanks the National Science Foundation for the Grant GP-812 which partially supported him during the preparation of this paper.

(2) If $M$ is a symmetric space of non-compact type, there will not exist such a group $L$, as is illustrated by the fact that the holomorphic transformation group of a bounded symmetric domain is contained in the isometry group. The proof will appear elsewhere. 
We wish here to remind the reader of an outstanding characteristic which is common to these examples. Let $M$ be one of the above spaces of compact type, and $M^{\prime}$ its noncompact form. Then there exists an isomorphism (with respect to the structure) of $M^{\prime}$ onto a bounded domain $D$ of the euclidean space of the same dimension; more precisely, the hyperbolic space can be diffeomorphically mapped onto an open ball $D$ in such a way that every geodesic is mapped onto a segment in $D$ [respectively, every angle between two vectors is preserved], and any hermitian space of noncompact type can be holomorphically imbedded onto a bounded domain $D$ (E. Cartan and Harish-Chandra). (When the dimension of $M$ is 2, the last two examples are nothing but the Poincaré realization.)

Now one may ask whether other symmetric spaces admit geometric transformation groups $L$, whether there are any common significant properties possessed by the pairs $(L, M)$, and what are the geometric structures with the automorphism groups $L$. This paper is to answer the first two questions, as follows.

If $M$ is the direct Riemann product of $M_{1}$ and $M_{2}$ and if each $M_{a}, a=1,2$, admits a geometric transformation group $L_{a}$, then the group $L=L_{1} \times L_{2}$ is naturally a geometric transformation group of $M=M_{1} \times M_{2}$. When this is the case, we say that (the operation of) $L$ on $M$ (and also $M$ ) is decomposable. So, in general, $L$ and $M$ are direct products of indecomposable factors. We should study indecomposable factors only. Roughly speaking $\left({ }^{3}\right)$, the fundamental theorem, which is the union of Theorems 3.1, 3.5 and 4.2, states the following (I) to (V): (I) if a compact symmetric space $M$ admits an indecomposable geometric transformation group $L$ and if the center of $G$ is of dimension $\leqq 1$, then $L$ is the isometry group of another symmetric space containing $M$, or, more precisely, $P=L / G$ is an irreducible symmetric space of noncompact type into which $M$ is $G$-equivariantly and isometrically imbedded. (II) Conversely, if an irreducible symmetric space $P=L / G$ of noncompact type has a $G$-orbit $M$ which is a symmetric space with respect to the induced Riemannian metric from $P$, then $L$ can act on $M$ so that all the assumptions of (I) are satisfied. (III) The operation of $L$ on $M$ mentioned in (I) is essentially unique. (IV) Under the assumptions of (I), the involutive automorphism of $G$ (which makes $M$ symmetric) extends to that of $L$, a simple group, and $F$, which denotes the group consisting of the elements left invariant by the extended automorphism of $L$, contains a one-dimensional vector group $Z$ in its center (or, equivalently, the linear isotropy group $F$ is reducible). (V) And, conversely, if $L$ is simple and $L / F$ is an affine symmetric space with reducible linear isotropy group $F$, then there exists (by Berger [2]) a maximal compact subgroup $G$ which is invariant under the involutive

(3) Indeed, we are confusing manifolds with their finite coverings and even with their connected components. Moreover, the assumption of indecomposability in (I) must be replaced by a slightly stronger one. We will begin with a much stronger assumpion in order to make proofs short following the referee's suggestion. The possibility of weakening the assumption will be discussed in the appendix. 
automorphism of $L$ and $L$ can act on $M=G / K, K=F \cap G$, so that the assumptions of (I) are satisfied.

This fundamental theorem will enable us to study the structure of the pair $(L, M)$ somewhat in detail. For instance, $L / F$ is $L$-equivariantly diffeomorphic with the cotangent bundle of $M$ on which $L$ acts naturally. In particular, in terms of any coordinate system, a transformation in $L$ is uniquely determined by its expression at any point with the third and higher derivatives neglected. Also we will examine the Lie algebra structure of $L$. If $\boldsymbol{L}, \boldsymbol{G}$, etc., denote the Lie algebras of $L, G$, etc. $\left({ }^{4}\right)$, under the assumptions of (I), and if $\boldsymbol{L}=\boldsymbol{G}+\boldsymbol{P}$ [respectively, $\boldsymbol{G}=\boldsymbol{K}+\boldsymbol{M}]$ is the Cartan decomposition for the symmetric space $L / G$ [respectively, $G / K=M]$, then there exists a nonzero element $\boldsymbol{z}$ in $\boldsymbol{P}$ with $[K, z]=0$. ( $z$ generates $Z$ in (IV).) This will imply the vector-space direct sum decomposition (the "Cartan decomposition" for the affine symmetric space $L / F$ ): $\boldsymbol{L}=\boldsymbol{M}_{+1}+\boldsymbol{M}_{-1}+\boldsymbol{F}$, where $\boldsymbol{M}_{e}=\{\boldsymbol{m}+e[z, \boldsymbol{m}] \mid \boldsymbol{m} \in \boldsymbol{M}\}, \quad e= \pm 1$, and $\boldsymbol{F}=\boldsymbol{K}+[[\boldsymbol{z}, \boldsymbol{M}], \boldsymbol{M}]$. Moreover, $\boldsymbol{M}_{e}$ and $\boldsymbol{F}$ are the eigenspaces of $\operatorname{ad}_{L} z$ corresponding to the eigenvalues $e$ and 0 . It will turn out that $F$ is the Lie algebra of $F$ and $M_{-1}+F$ is that of $H$, the isotropy subgroup for $L$ acting on $M$ (a part of Theorem 4.6). So the structure of $L$ is quite similar to that of the holomorphic transformation group of a compact hermitian space. In this special case $i z, i^{2}=-1$, belongs to the center of $K$, giving rise to the complex structure. Another example to illustrate the Lie algebra structure stated above. Let $M=G / K$ be the Grassmann manifold $\mathrm{SO}(m) / \mathrm{SO}(p) \times \mathrm{SO}(q), m=p+q, p q>0 . G$ is the automorphism group of the oriented real $m$-dimensional metric vector space, which we here denote by $U$. The symmetric space $M$ is interpreted as the space of $p$-dimensional oriented subspaces of $U$. Let $V$ be one of those subspaces, and $W$ its orthogonal complement. $K$ is then interpreted as the subgroup of $G$ consisting of the automorphisms of $U$ which leave $V$ invariant (hence $W$ also). If $L$ denotes the special linear group $\operatorname{SL}(m, R)$ of $U, L$ is an automorphism group of $U$ with the metric disregarded and its Lie algebra $L$ consists of the linear mappings of $U$ into itself with vanishing trace. The elements of $L$ send the oriented subspaces of $U$ onto the oriented subspaces. In particular, $L$ acts on $M$, containing $G$ properly. The operation is effective. But we find it convenient to consider $\boldsymbol{L}$ as a space of linear operators on $U$ in order to explain the Lie algebra structure of $\boldsymbol{L}$. $\boldsymbol{G}$ consists of the skew-symmetric operators in $\boldsymbol{L}$, while the symmetric operators in $\boldsymbol{L}$ form the space $\boldsymbol{P}$ in the Cartan decomposition of $\boldsymbol{L}$. The element $\boldsymbol{z}$ of $\boldsymbol{P}$ is defined by $z(x+y)=a x+b y$ for $x \in V$ and $y \in W$, where $a, b$ are scalars determined by $p a+q b=0$ and $a-b=1$. The space $M$ in the Cartan decomposition of $\boldsymbol{G}$ with respect to the symmetric-space structure of $M$ consists of the operators $\boldsymbol{f}$ in $\boldsymbol{G}$ with the properties $f(V) \subset W$ and $f(W) \subset V . M_{+1}$ consists of the operators

(4) Always we will denote the Lie algebras and their subspaces by (the corresponding) boldface letters. 
$\boldsymbol{f}$ in $\boldsymbol{L}$ with $\boldsymbol{f}(U) \subset W$ and $f(W)=0, M_{-1}$ of $\boldsymbol{f}$ with $f(U) \subset V$ and $f(V)=0$, the subalgebra $\boldsymbol{F}$ of $\boldsymbol{f}$ with $\boldsymbol{f}(V) \subset V$ and $\boldsymbol{f}(W) \subset W$, and $\boldsymbol{H}$ of $\boldsymbol{f}$ with $\boldsymbol{f}(V) \subset V$.

Furthermore, the classification of all the (local) pairs $(L, M)$ can be formulated in two ways by means of the two parts (I) + (II) and (IV) + (V) of the fundamental theorem. As an application we shall prove the generalization of the realization mentioned above by defining an "isomorphism" of the noncompact form $M^{\prime}$ of $M$ into $M$ and using Harish-Chandra's method for the hermitian case. As the reader may suspect from the part (I) $+($ II),$(L, M)$ has some connection with the theory of spherical functions (in the framework of E. Cartan), hence with elliptic differential equations. In fact, $z$ denoting the vector field on $M$ which generates $Z$, there exists a real spherical function $f$ on $M$ such that its exterior differential, $d f$, is identified with $z$ by the Riemannian metric. (In the imbedding of $M$ into $P$, the origin of $M$ is sent to expz.) As another application of the fundamental theorem, it will be shown that the function $f$ has nice properties from the viewpoint of the Bott-Morse theory. Other applications will be published in subsequent papers under joint authorship with S. Kobayashi.

Since the proof of the fundamental theorem is rather complicated, we put the Lie algebra arguments in $\S 1$. (The postulates (P.1-5) there on which they are based will turn out to characterize the infinitesimal situation of $(L, M)$.) $\$ 2$ is the first half of the demonstration of the fundamental theorem, which will be completed in $\$ 3 . \S 4$ is devoted to the structure theorems. In $\$ 5$ we will classify all the pairs $(L, M)$. The list at the end of this section indicates a number of imbeddings of compact symmetric spaces into others obtained with the classification together with (I). Some of them were used successfully by several authors. The last two sections, 6 and 7, are for the two applications.

We refer the reader almost exclusively to [4] for the necessary knowledge and the original papers to which we owe much.

Finally we express our profound gratitude to N. Tanaka (Nagoya University) for many valuable suggestions and conjectures which have been indispensable for our study.

\section{Preliminary lemmas.}

Definition. Given a Lie algebra $\boldsymbol{G}$ and an automorphism $\alpha$ of $\boldsymbol{G},(\boldsymbol{G}, \alpha)$ is called a symmetric pair when $\alpha$ is involutive and $\alpha$ is different from the identity. $(\boldsymbol{G}, \alpha)$ is said to be compact when some $G$ generated by $\boldsymbol{G}$ is compact.

Definition. A homogeneous manifold $M=G / K$, on which $G$ is almost effective, is called a Riemannian [respectively, affine] symmetric space by a symmetric pair $(G, \alpha)$ when (1) $\alpha$ is induced from an automorphism of $G,(2)$ $\boldsymbol{G}_{\alpha}=\boldsymbol{K}$, and (3) $K^{\prime}$ is compact [respectively, noncompact], where $\boldsymbol{G}_{\alpha}$ is the set of fixed points of $\alpha$ and $K^{\prime}$ is $K$ modulo (the transformations which act trivially on $M)$. As regards symmetric pair and space, we refer the reader to Helgason [4]. A symmetric pair $(\boldsymbol{G}, \alpha)$ gives rise to the so-called Cartan decomposition: 
$\boldsymbol{G}=\boldsymbol{G}_{\alpha}+\boldsymbol{G}_{-a}$, where $\boldsymbol{G}_{-\alpha}$ is the eigenspace of $\alpha$ corresponding to -1 . So we have $\left[\boldsymbol{G}_{\alpha}, \boldsymbol{G}_{\alpha}\right] \subset \boldsymbol{G}_{\alpha},\left[\boldsymbol{G}_{\alpha}, \boldsymbol{G}_{-\alpha}\right] \subset \boldsymbol{G}_{-\alpha}$ and $\left[\boldsymbol{G}_{-\alpha}, \boldsymbol{G}_{-\alpha}\right] \subset \boldsymbol{G}_{\alpha}$.

The following postulates (P. 1-5) are fundamental in this section.

(P. 1) $(\boldsymbol{G}, \alpha)$ and $(\boldsymbol{L}, \beta)$ are symmetric pairs.

(P. 2) L is simple.

(P. 3) $G=L_{\beta}$.

(P. 4) $\boldsymbol{G}$ is a maximal compactly imbedded [4] subalgebra of $\boldsymbol{L}$.

(P. 5) There exists an element $z(\neq 0)$ in $L_{-\beta}$ with $\left[G_{\alpha}, z\right]=\{0\}$.

The Killing form $B=B_{L}$ of $L$ is positive [respectively, negative] definite if it is restricted to $L_{-\beta}\left[\right.$ respectively, $\left.L_{\beta}\right]$.

$$
\begin{aligned}
& {[z, Q]=\{0\} \text {, where } \boldsymbol{Q} \text { denotes the orthogonal complement }} \\
& \text { of }\left[z, G_{-\alpha}\right] \text { in } L_{-\beta} \text { with respect to } B .
\end{aligned}
$$

Since $[z, Q]$ is contained in $\boldsymbol{G},(1.1)$ follows from

$$
B([z, Q], G)=B(Q,[z, G])=B\left(Q,\left[z, G_{-\alpha}\right]\right)=0,
$$

the second equality being due to (P. 5).

$$
\left[z, G_{-\alpha}\right] \text { has the same dimension as } \boldsymbol{G}_{-\alpha} \text {. }
$$

Let $\boldsymbol{M}_{1}$ be the kernel of $\operatorname{ad} z \mid \boldsymbol{G}_{-\alpha}$. We have to show $\boldsymbol{M}_{1}=\{0\} . \quad\left[\boldsymbol{G}_{\alpha}, \boldsymbol{M}_{1}\right]$ is a subspace of $\boldsymbol{M}_{1}$ by (P. 5). $\boldsymbol{M}_{2}$ denoting the orthogonal complement of $\boldsymbol{M}_{1}$ in $G_{-\alpha}$ with respect to $B$, we have $\left[M_{2}, M_{1}\right]=\{0\}$ owing to the fact that $B\left(\left[\boldsymbol{M}_{2}, \boldsymbol{M}_{1}\right], \boldsymbol{G}_{\alpha}\right)=B\left(\boldsymbol{M}_{2},\left[\boldsymbol{M}_{1}, \boldsymbol{G}_{\alpha}\right]\right) \subset B\left(\boldsymbol{M}_{2}, \boldsymbol{M}_{1}\right)=\{0\}$. Thus $\left[\boldsymbol{G}_{-\alpha}, \boldsymbol{M}_{1}\right]$ is contained in $\left[\boldsymbol{M}_{1}, \boldsymbol{M}_{1}\right]$. Hence $\boldsymbol{G}_{1}=\left[\boldsymbol{M}_{1}, \boldsymbol{M}_{1}\right]+\boldsymbol{M}_{\mathbf{1}}$ is an ideal in $\boldsymbol{G}$. Let $\boldsymbol{P}_{\mathbf{1}}$ denote the orthogonal complement in $L_{-\beta}$ of the space spanned by $z$. By (P. 4) and $\left[z, G_{1}\right]=\{0\}$, we have $\left[G_{1}, P_{1}\right] \subset P_{1}$. Naturally, $L_{-\beta}$ is a $G$-module which is simple by (P. 1-4). On the other hand, $\left[G_{1}, L_{-\beta}\right]$ is a sub-G-module. By $\left[G_{1}, P_{1}\right] \subset P_{1}$ and $\left[G_{1}, z\right]=\{0\},\left[G_{1}, L_{-\beta}\right]$ cannot contain $z$, hence it equals $\{0\}$. Thus $G_{1}$ is a proper ideal in $L$, which gives $M_{1} \subset G_{1}=\{0\}$ by (P. 2). (1.2) is thus proved.

$$
\left[z,\left[z, G_{-\alpha}\right]\right]=G_{-\alpha} .
$$

The left-hand side is contained in $\boldsymbol{G}$. Moreover, it is contained in $\boldsymbol{G}_{-\alpha}$ because of $B\left(\left[z,\left[z, G_{-\alpha}\right]\right], G_{\alpha}\right)=B\left(\left[z, G_{-\alpha}\right],\left[z, G_{\alpha}\right]\right)=\{0\}$ which is due to (P.5). Let $B^{\prime}$ be a symmetric bilinear form on $\boldsymbol{G}_{-\alpha}$ defined by

$$
\left.B^{\prime}\left(m_{1}, m_{2}\right)=B\left(\left[z,\left[z, m_{1}\right]\right], m_{2}\right)\right)=-B\left(\left[z, m_{1}\right],\left[z, m_{2}\right]\right)
$$

for any $m_{1}, m_{2}$ in $G_{-\alpha} . B^{\prime}$ is negative definite by (1.2). (1.3) thus follows.

$$
\left[\left[z, G_{-\alpha}\right],\left[z, G_{-\alpha}\right]\right] \subset G_{\alpha} .
$$

In view of $\left[\boldsymbol{G}_{-\alpha}, \boldsymbol{G}_{-\alpha}\right] \subset \boldsymbol{G}_{\alpha},\left(\right.$ P. 5) implies $\left[z,\left[z,\left[\boldsymbol{G}_{-\alpha}, \boldsymbol{G}_{-\alpha}\right]\right]\right]=0$. The left- 
hand side equals $\left[\left[z,\left[z, G_{-\alpha}\right]\right], G_{-\alpha}\right]+\left[\left[z, G_{-\alpha}\right],\left[z, G_{-\alpha}\right]\right]$, while the first is contained in $G_{\alpha}$ by (1.3).

LEMMA 1.1. Under the assumptions (P. 1-5), the automorphism $\alpha$ of $\boldsymbol{G}$ extends to an involutive one, also denoted by $\alpha$, of $\boldsymbol{L}$ so that $\boldsymbol{L}_{\alpha}=\boldsymbol{G}_{\alpha}+\boldsymbol{Q}$ and $\boldsymbol{L}_{-\alpha}=\boldsymbol{G}_{-\alpha}+\left[z, \boldsymbol{G}_{-\alpha}\right]$, where $\boldsymbol{Q}$ is the orthogonal complement of $\left[\mathbf{z}, \boldsymbol{G}_{-\alpha}\right]$ in $L_{-\beta}$ with respect to $B$.

The lemma will be true if we can establish the following three assertions:

The space $\boldsymbol{F}=\boldsymbol{G}_{\alpha}+\boldsymbol{Q}$ is a subalgebra.

$$
\left[\boldsymbol{F}, \boldsymbol{G}_{-\alpha}+\left[z, G_{-\alpha}\right]\right] \subset \boldsymbol{G}_{-\alpha}+\left[z, G_{-\alpha}\right] \text {. }
$$$$
\left[\boldsymbol{G}_{-\alpha}+\left[z, \boldsymbol{G}_{-\alpha}\right], \boldsymbol{G}_{-\alpha}+\left[z, \boldsymbol{G}_{-\alpha}\right]\right] \subset \boldsymbol{F} .
$$

To verify (1.5), it is sufficient to see

$$
\boldsymbol{F} \text { coincides with the centralizer } \boldsymbol{C} \text { of } z \text { in } \boldsymbol{L} \text {. }
$$

In fact, $C$ contains $\boldsymbol{F}$ by (P. 5) and (1.1). And $\boldsymbol{C}$ is contained in $\boldsymbol{F}$; otherwise there would exist $\boldsymbol{m}_{1}, \boldsymbol{m}_{2}$ in $\boldsymbol{G}_{-\alpha}$ with $\left[z, \boldsymbol{m}_{1}+\left[z, \boldsymbol{m}_{2}\right]\right]=0$, and $\boldsymbol{m}_{1}+\left[z, \boldsymbol{m}_{2}\right] \neq 0$, which contradicts to (1.2) and (1.3), owing to the facts $\left[z,\left[z, m_{2}\right]\right] \in G_{-\alpha}$ and $\left[z, G_{-\alpha}\right] \cap \boldsymbol{G}_{-\alpha}=\{0\} .(1.5)$ is proved.

(P. 5) clearly implies $\left[\boldsymbol{G}_{\alpha}, \boldsymbol{G}_{-\alpha}+\left[z, \boldsymbol{G}_{-\alpha}\right]\right] \subset \boldsymbol{G}_{-\alpha}+\left[z, \boldsymbol{G}_{-\alpha}\right]$. Thus (1.6) follows from

$$
\left[Q, G_{-\alpha}\right] \subset\left[z, G_{-\alpha}\right],\left[Q,\left[z, G_{-\alpha}\right]\right] \subset G_{-\alpha} .
$$

To prove (1.9), let $B$ denote the Killing form of $L$ again. Since $\left[\boldsymbol{Q}, \boldsymbol{G}_{-\alpha}\right]$ is contained in $L_{-\beta}, B\left(\left[\boldsymbol{Q}, \boldsymbol{G}_{-\alpha}\right], \boldsymbol{Q}\right)=B\left(\boldsymbol{G}_{-\alpha},[\boldsymbol{Q}, \boldsymbol{Q}]\right) \subset B\left(\boldsymbol{G}_{-\alpha}, \boldsymbol{C} \cap \boldsymbol{G}_{\alpha}\right)=B\left(\boldsymbol{G}_{-\alpha}, \boldsymbol{G}_{\alpha}\right)$ $=\{0\}$ by (1.8) and (1.3). Hence $\left[Q, G_{-\alpha}\right] \subset\left[z, G_{-\alpha}\right]$ in view of the definition of $\boldsymbol{Q}$. This fact and (1.8) combined with (1.3) give that

$$
\left[Q,\left[z, G_{-\alpha}\right]\right]=\left[z,\left[Q, G_{-\alpha}\right]\right] \subset\left[z,\left[z, G_{-\alpha}\right]\right]=G_{-\alpha} .
$$

Finally, to obtain (1.7), by (1.4), we have only to see

$$
\left[\boldsymbol{G}_{-\alpha},\left[z, G_{-\alpha}\right]\right] \subset Q .
$$

This is, however, obvious from $B\left(\left[\boldsymbol{G}_{-\alpha},\left[z, \boldsymbol{G}_{-\alpha}\right]\right],\left[z, \boldsymbol{G}_{-\alpha}\right]\right)=B\left(\left[\boldsymbol{G}_{-\alpha},\left[z, \boldsymbol{G}_{-\alpha}\right]\right.\right.$, $\left.\left.\left[z, \boldsymbol{G}_{-\alpha}\right]\right]\right) \subset B\left(\boldsymbol{G}_{-\alpha}, \boldsymbol{G}_{\alpha}\right)=\{0\}$ by (1.4).

Extending $\alpha$ to an automorphism of $L$, we have obtained a symmetric pair $(\boldsymbol{L}, \alpha)$ (Lemma 1.1). Thus $\alpha$ and $\beta$ commute on $\boldsymbol{L}$. The automorphism $\alpha$ of $\boldsymbol{L}$ extends to an involutive one, also denoted by $\alpha$, of the complexification of $L$. $\alpha$ leaves invariant its real subalgebra $\boldsymbol{L}_{u}=\left\{a+i \boldsymbol{b} \mid \boldsymbol{a} \in \boldsymbol{L}_{\beta}, \boldsymbol{b} \in \boldsymbol{L}_{-\beta}\right\}, i^{2}=-1$, and induces on it an involutive automorphism, again denoted by $\alpha$.

LEMMA 1.2. Under the postulates (P. 1-5), the above-defined symmetric 
pair $\left(\boldsymbol{L}_{u}, \alpha\right)$ is compact and hermitian (see the proof). Moreover, either $\boldsymbol{L}_{\boldsymbol{u}}$ is simple, or $\boldsymbol{G}$ is simple and $(\boldsymbol{G}, \alpha)$ is hermitian.

Proof. We have $\left(\boldsymbol{L}_{u}\right)_{\alpha}=\boldsymbol{G}_{\alpha}+i \boldsymbol{Q}, \boldsymbol{Q}$ as in Lemma 1.1 , and $\left(\boldsymbol{L}_{\boldsymbol{u}}\right)_{-\alpha}=\boldsymbol{G}_{-\alpha}$ $+i\left[z, G_{-\alpha}\right]$. The center of $\left(\boldsymbol{L}_{\boldsymbol{u}}\right)_{\alpha}$ contains a nonzero element $i \boldsymbol{z}$. In this sense $\left(\boldsymbol{L}_{u}, \alpha\right)$ is hermitian. By (P. 2), $\boldsymbol{L}_{\boldsymbol{u}}$ is semisimple. The Killing form of $\boldsymbol{L}_{\boldsymbol{u}}$ is negative definite, and thus $\left(\boldsymbol{L}_{u}, \alpha\right)$ is compact. Suppose $\boldsymbol{L}_{\boldsymbol{u}}$ is not simple. $\boldsymbol{L}_{\boldsymbol{u}}$ is then isomorphic with $\boldsymbol{G} \times \boldsymbol{G}$ and $\boldsymbol{G}$ is simple. In this sense, $(\boldsymbol{G}, \alpha)$ is irreducible. $\boldsymbol{L}$ can be identified with the complexification $\boldsymbol{G}+j \boldsymbol{G}, j^{2}=-1$. Since $z$ belongs to $\boldsymbol{L}_{-\beta}=j \boldsymbol{G}, j \boldsymbol{z}$ belongs to $\boldsymbol{G}$, which is simple, and to $\boldsymbol{G}_{\alpha}$ by (P. 5). Therefore $\boldsymbol{G}_{\alpha}$ contains $j z$ in its center. Hence, $(G, \alpha)$ is hermitian.

LEMmA 1.3. Under the hypotheses (P. 1-5), $z$ in (P. 5) can be so chosen that

(1) restricted to $\boldsymbol{G}_{-\alpha},(\mathrm{adz})^{2}$ is the identity,

(2) $\left[\left[z, m_{1}\right],\left[z, m_{2}\right]\right]=-\left[\boldsymbol{m}_{1}, \boldsymbol{m}_{2}\right]$ for any $\boldsymbol{m}_{1}, \boldsymbol{m}_{2}$ in $\boldsymbol{G}_{-\alpha}$,

(3) the space $\boldsymbol{M}_{\boldsymbol{e}}=\left\{\boldsymbol{m}+e[z, \boldsymbol{m}] \mid \boldsymbol{m} \in \boldsymbol{G}_{-\alpha}\right\}, e=1$ or -1 , is an abelian subalgebra of $\boldsymbol{L}$.

Proof. We preserve the notations in the proof of Lemma 1.2. In case $\boldsymbol{L}_{\boldsymbol{u}}$ is simple, $\left(\boldsymbol{L}_{u}, \alpha\right)$ is hermitian and therefore there exists a real number $c$ such that $(\operatorname{ad} i c z)^{2}$ is -(identity) on $\left(\boldsymbol{L}_{u}\right)_{-\alpha}=\boldsymbol{G}_{-\alpha}+i\left[z, \boldsymbol{G}_{-\alpha}\right]$. In case $\boldsymbol{L}_{u}$ is not simple, $\boldsymbol{G}$ is simple by Lemma 1.2, and therefore there exists a real number $c$ such that $(\operatorname{adj} c z)^{2}$ is -(identity) on $\boldsymbol{L}_{-\beta}=j \boldsymbol{G}$. In both cases, we replace $z$ by $c z$ and obtain (1). Hereafter $c z$ shall be denoted by $z$. Then (2) immediately follows from $0=\left[z,\left[z,\left[m_{1}, m_{2}\right]\right]\right]=\left[\left[z,\left[z, m_{1}\right]\right], m_{2}\right]+2\left[\left[z, m_{1}\right],\left[z, m_{2}\right]\right]+\left[m_{1},\left[z,\left[z, m_{2}\right]\right]\right]$.

(3) is readily derived from (2) and (P. 5).

LEMMA 1.4. The conditions (P. 1-5) imply:

(1) $\boldsymbol{H}=\boldsymbol{F}+\boldsymbol{M}_{-1}$, where $\boldsymbol{F}=\boldsymbol{G}_{\alpha}+\boldsymbol{Q}$, is the normalizer, $\boldsymbol{N}$, of $\boldsymbol{M}_{-1}$ in $\boldsymbol{L}, \boldsymbol{Q}$ and $\boldsymbol{M}_{-1}$ being as in Lemmas 1.1 and 1.3.

(2) The adjoint representation, ad $\boldsymbol{F}$, of $\boldsymbol{L}$ restricted to $\boldsymbol{F}$, leaves $\boldsymbol{M}_{e}, e=1$ or -1, invariant and operates on it irreducibly.

(3) $\boldsymbol{H}$ is a maximal subalgebra of $\boldsymbol{L}$.

Proof. ad $\boldsymbol{F}$ leaves $\boldsymbol{M}_{+1}, \boldsymbol{M}_{-1}$ invariant by (1.9), (1) of Lemma 1.3, and (P. 5). From (3) of Lemma 1.3, it thus follows that $\boldsymbol{H}$ is contained in $\boldsymbol{N}$. To prove (2), assume that ad $\boldsymbol{F}$ is reducible on $\boldsymbol{M}_{+1}$ or $\boldsymbol{M}_{-1}$. Then there exists a nontrivial invariant subspace $\boldsymbol{M}^{\prime}$ of $\boldsymbol{G}_{-\alpha}$ such that $\left\{\boldsymbol{m}+e[\boldsymbol{z}, \boldsymbol{m}] \mid \boldsymbol{m} \in \boldsymbol{M}^{\prime}\right\}$ is a nontrivial invariant subspace of $\boldsymbol{M}_{\boldsymbol{e}}$. Also, since ad $\boldsymbol{K}$ is reducible on $\boldsymbol{M}, \boldsymbol{G}$ is not simple and $\boldsymbol{L}_{\boldsymbol{u}}$ in Lemma 1.2 is simple. Hence $\operatorname{ad}\left(\boldsymbol{L}_{\boldsymbol{u}}\right)_{\alpha}$ is irreducible on $\left(\boldsymbol{L}_{\boldsymbol{u}}\right)_{-\alpha}$. On the other hand, $\boldsymbol{M}^{\prime}+i\left[\boldsymbol{z}, \boldsymbol{M}^{\prime}\right]$ is a nontrivial invariant subspace of $\operatorname{ad}\left(\boldsymbol{L}_{\boldsymbol{u}}\right)_{\alpha}$. This contradiction proves (2). By $\boldsymbol{L}=\boldsymbol{H}+\boldsymbol{M}_{+1}$, (2) implies that $\boldsymbol{H}$ is a maximal 
subalgebra of $L$, and (3) is proved. Since $L$ is simple by (P. 2), the subalgebra $N$ containing $\boldsymbol{H}$ does not coincide with $\boldsymbol{L}$. Therefore (3) gives that $\boldsymbol{H}$ is $\boldsymbol{N}$, and Lemma 1.4 is proved.

2. Necessary conditions. Throughout this section, we shall assume the following four conditions (A. 1-4) satisfied:

(A. 1) $M=G / K$ is a connected compact symmetric space by a symmetric pair $(\boldsymbol{G}, \alpha)$ on which the connected isometry group $G$ operates effectively.

(A. 2) A connected Lie transformation group L operates on $M$ effectively and $L$ properly (namely, $\neq G$ ) contains $G$ as a transformation group of $M$, so that $G$ is a Lie subgroup of $L$.

(A. 3) The center of $G$ is of dimension $\leqq 1$, and

(A. 4) $L$ is simple.

Actually the assumption (A.3) is redundant; in fact, (A.3) will follow from the other assumptions (A.1,2 and 4) in view of Lemma 2.1 below. But we like to keep it here in order to refer to it in the appendix where (A.4) will not be assumed. All the lemmas and other assertions will be stated without mentioning (A.1-4), but with them kept in mind in this section.

LEMMA 2.1. $G$ is a maximal compact subgroup of $L$.

Proof. Let $G^{\prime}$ be a maximal compact subgroup of $L$ which contains $G$. $G^{\prime}$ leaves invariant some Riemannian metric $\gamma$ on $M$. $M$ is necessarily a symmetric space with respect to $\gamma$, since $\gamma$ is invariant under $G$. By compactness of $G$, it follows that $G$ is the connected component of the isometry group of $\gamma$. Thus $G$ contains $G^{\prime}$, and $G=G^{\prime}$.

LEMMA 2.2. The center $C$ of $L$ is finite.

Proof. Let $C(G)$ be the centralizer of $G$ in the group of all differentiable transformations of $M . C(G)$ contains $C . C(G)$ is isomorphic with $N(K) / K$, where $N(K)$ is the normalizer of $K$ in $G[6] . N(K) / K$ naturally contains the identity component $G_{0}$ of the center of $G$, and an infinite subgroup of $N(K) / K$ has an infinite number of elements in common with $G_{0}$ by a property of a compact symmetric space $G / K$. If $C$ is infinite, $C \cap G_{0}$ is therefore infinite, in particular, $G_{0}$ is a circle group by (A.3). Since $C \cap G_{0}$ is closed in $G$, it follows that $C$ contains $G_{0}$, contrary to Lemma 2.2 .

For later use in the appendix we note that Lemma 2.2 remains true if $L$ is semisimple instead of (A.4).

Lemmas 2.1 and 2.2 immediately give

LEMMA 2.3. L/G is naturally an irreducible symmetric space of noncompact type.

$L$ is almost effective on $L / G$ by Lemma 2.4 . 
COROLlary 2.3. $G$ is a maximal connected Lie subgroup of $L$.

If $M$ is oriented, a volume element $\omega$ on $M$ is uniquely determined by a $G$-invariant Riemannian metric on $M$. We denote by $V$ the group of differentiable transformations of $M$ which leave $\omega$ invariant.

LEMMA 2.4. Lis not contained in the volume-preserving group $V\left({ }^{5}\right)$. Hence $G$ is the set of elements in L leaving $\omega$ invariant.

Proof. Let $\mathscr{F}$ be the set of all complex-valued differentiable functions on $M$. $\mathscr{F}$ is naturally a vector space over the complex field. For two functions $f_{1}, f_{2}$ in $\mathscr{F}$, their inner product $\left(f_{1}, f_{2}\right)$ is defined by $\int_{M} f_{1} \bar{f}_{2} \omega$, where $\bar{f}_{2}$ is the complex conjugate of $f_{2}$. Any differentiable transformation $t$ of $M$ operates linearly on $M$ by $t(f)=f \circ t^{-1}$ for each $f$ in $\mathscr{F}$. If $t$ leaves $\omega$ invariant, $t$ is unitary in the sense that the inner product in $\mathscr{F}$ is invariant under $t$. Thus $G$ is a unitary transformation group of $\mathscr{F}$. E. Cartan [3] showed that $\mathscr{F}$ is the direct sum of finitedimensional subspaces, $\mathscr{F}_{\lambda}$, on which $G$ operates irreducibly, and that the representations of $G$ on different spaces $\mathscr{F}_{\lambda}$ and $\mathscr{F}_{\mu}$ are not equivalent.

By Lemma 2.3, $L$ admits an involutive automorphism $\beta$ such that $(L, \beta)$ is a symmetric pair with $\boldsymbol{L}_{\beta}=\boldsymbol{G}$ in the notations of $\S 1$. Let $\boldsymbol{P}$ denote $\boldsymbol{L}_{-\beta}$. We consider $\boldsymbol{L}$ as a set of vector fields on $M$. We have $[\boldsymbol{G}, \boldsymbol{P}] \subset \boldsymbol{P}$ and $[\boldsymbol{P}, \boldsymbol{P}] \subset \boldsymbol{G}$. The former implies that

$$
\text { the space } \boldsymbol{P} \mathscr{F}_{\lambda}=\left\{\boldsymbol{p} f \mid \boldsymbol{p} \in \boldsymbol{P}, f \in \mathscr{F}_{\lambda}\right\} \subset \mathscr{F} \text { is G-invariant. }
$$

From the above theorem of E. Cartan follows

$$
\begin{gathered}
\boldsymbol{P} \mathscr{F}_{\lambda} \text { is the sum of some finite number of } \mathscr{F}_{\mu} \text { 's, } \\
\qquad \boldsymbol{p}_{1} \boldsymbol{p}_{2} f=\boldsymbol{p}_{2} \boldsymbol{p}_{1} f \bmod \mathscr{F}_{\lambda} \text { for } \boldsymbol{p}_{1}, \boldsymbol{p}_{2} \in \boldsymbol{P}, f \in \mathscr{F}_{\lambda} .
\end{gathered}
$$

This is a consequence of the relation $[P, P] \subset G$. Let $\boldsymbol{T}$ be a Cartan subalgebra of $\boldsymbol{G} . f_{\boldsymbol{\theta}, \lambda}\left[\right.$ respectively, $\left.\boldsymbol{p}_{\alpha}\right]$ shall denote an arbitrary weight factor, in the space of $\mathscr{F}_{\lambda}$ [respectively, $\boldsymbol{G}$-module $\boldsymbol{P} \otimes \boldsymbol{C}=$ the complexification of $\left.\boldsymbol{P}\right]$, corresponding to the weight $\theta$ [respectively, $\alpha]$ with respect to $\boldsymbol{T}$. Then we have immediately that

$$
\left(f_{\theta, \lambda}, f_{\phi, \mu}\right) \neq 0 \text { implies } \theta=\phi, \lambda=\mu \text {, }
$$

and

$$
\begin{aligned}
& \boldsymbol{p}_{\alpha} f_{\theta, \lambda} \text { is a weight vector corresponding to } \\
& \text { the weight } \alpha+\theta \text { (if it is different from zero). }
\end{aligned}
$$

Now we assume that Lemma 2.4 is false and that $L$ is a subgroup of $V$. Then each $\boldsymbol{p}_{\alpha}$ is skew-hermitian.

(5) This makes no sense if $M$ is not orientable. In that case, we consider an orientable covering space of $M$ instead of $M$ which will clearly satisfy (A. 1-4). This will be no loss of generality for the main results. 


$$
\text { If } \boldsymbol{p}_{\alpha} p_{\beta} f_{\theta, \lambda} \text { does not belong to } \mathscr{F}_{\lambda} \text {, then } \alpha=\beta=0 \text {. }
$$

In fact, then there exists some $f_{\phi, \mu}$ such that $\left(\boldsymbol{p}_{\alpha} \boldsymbol{p}_{\beta} f_{\theta, \lambda}, f_{\phi, \mu}\right) \neq 0$. Hence, $\phi$ equals $\alpha+\beta+\theta$ by (2.5). Since $\boldsymbol{p}_{\alpha}$ is skew-hermitian, it also follows that $\left(\boldsymbol{p}_{\beta} f_{\theta, \lambda}, \boldsymbol{p}_{\alpha} f_{\phi, \mu}\right) \neq 0$. By (2.4) and (2.5), we thus obtain $\beta+\theta=\alpha+\phi$, whence $\beta+\theta=\alpha+(\alpha+\beta+\theta)$ and so $\alpha=0$. We also get $\beta=0$ by (2.3), and (2.6) is proved.

From (2.6), we infer that the weights of the representation of $G$ on $\boldsymbol{P P} \mathscr{F}_{\lambda}$ are those for $\mathscr{F}_{\lambda}$. Hence $\boldsymbol{P P} \mathscr{F}_{\lambda}$ is contained in $\mathscr{F}_{\lambda}$ if $\mathscr{F}_{\lambda}$ is so chosen that the highest weight for $\mathscr{F}_{\lambda}$ is the lowest among the highest weights for all $\mathscr{F}_{\mu} \neq \mathscr{F}_{0}$. $\mathscr{F}_{\lambda}$ being chosen as above, the space $\mathscr{F}_{\lambda}+\boldsymbol{P} \mathscr{F}_{\lambda}$ is, therefore, invariant under $\boldsymbol{L}=\boldsymbol{G}+\boldsymbol{P}$, therefore under $L$. In other words, the simple group $L$ operates nontrivially on a finite-dimensional vector space as a unitary group. $L$ is thus compact, contrary to Lemma 2.1. The proof of Lemma 2.4 is completed.

Consider the mapping $\boldsymbol{p} \rightarrow \operatorname{div}(\boldsymbol{p})$ of $\boldsymbol{P}$ into $\mathscr{F}$. The Lie derivative $\theta(\boldsymbol{p})$ satisfies $\theta(\boldsymbol{p}) \omega=(\operatorname{div} \boldsymbol{p}) \omega$ and $\theta(\phi(\boldsymbol{p}))=\phi \theta(\boldsymbol{p}) \phi^{-1}$ if $\phi$ is a diffeomorphism of $M$ onto itself and also denotes the automorphism caused by $\phi$ of the space of all the tensor fields on $M$. If $\phi$ belongs to $G$ then it follows that $\operatorname{div}(\phi(p))=(\operatorname{div} p) \circ \phi$, so the mapping is a $G$-module homomorphism which, by Lemma 2.4 , does not map $\boldsymbol{P}$ into 0 . Also $\int(\operatorname{div} \boldsymbol{p}) \omega=0$, so $\boldsymbol{P}$ is isomorphic as a $G$-module to the real form of some $\mathscr{F}_{\lambda}$. Thus we have $\operatorname{proved}\left({ }^{6}\right)$

LEMMA 2.5. The natural (=adjoint) representation of $G$ on $\boldsymbol{P}$ is of class 1 .

This lemma is known (E. Cartan [3]) to be equivalent to the condition that there exists a nonzero element $z$ in $\boldsymbol{P}$ which is left invariant by $K$, the isotropy subgroup of $G ; \operatorname{ad}_{L}(k) z=z$ for each $k$ in $K$. If $(G, \alpha)$ denotes the symmetric pair corresponding to the symmetric structure of $M=G / K$, then the Lie algebra $K$ of $K$ is $\boldsymbol{G}_{\alpha}$, in the notation at the beginning of $\S 1 . L$ admits an involutive automorphism $\beta$ with $\boldsymbol{G}=\boldsymbol{L}_{\boldsymbol{\beta}}$ by Lemma 2.3. From the assumptions (A.1-4) of this section, we have thus deduced the postulates (P. 1-5) at the beginning of $\S 1$; in fact, (P. 1) is implied by (A. 1) and Lemma 2.3, (P. 4) by Lemma 2.1, (P. 5) by the above (or Lemma 2.5), and all those lemmas have followed from (A. 1-4).

3. The main theorem. Let $\mathscr{A}$ be the set of all the triple systems $(G, K, L)$ with $M=G / K$ and $L$ satisfying(A. 1-4) stated at the beginning of $\S 2$. Let $\mathscr{P}$ be the set of all the pairs $(M, P)$ of compact locally symmetric Riemannian homogeneous manifolds $M=G / K$ and irreducible Riemannian symmetric spaces $P=L / G$ of noncompact type with $L$ connected and effective on $P$ such that $M$ is a $G$-orbit ( $\neq$ one point) in $P$.

THEOREM 3.1. In these notations, there exists a mapping $\Phi$ of $\mathscr{A}$ onto $\mathscr{P}$ such that $\left(M^{\prime}, P\right)$ denoting $\Phi(G, K, L),(1)$ there exists a subgroup $K^{\prime}(\supset K)$

(6) We owe this simple proof to the referee. We are grateful to him for it and many other valuable suggestions. 
of $G$ with finite index $\left[K^{\prime}: K\right]$ and with $M^{\prime}=G / K^{\prime}$, (2) $P=L / G$. (If ad denotes the homomorphism of $L$ onto its adjoint group, then $\operatorname{ad}(L)$ operates on $P^{\prime}$ effectively, $P^{\prime}=\operatorname{ad}(L) / \operatorname{ad}(G)$ and $M^{\prime}=\operatorname{ad}(G) / \operatorname{ad}\left(K^{\prime}\right)$.)

Proof. For any element $(G, K, L)$ in $\mathscr{A}$, the space $P=L / G$ is an irreducible symmetric space of noncompact type (Lemma 2.3). The adjoint group $L^{\prime}$ operates effectively on $P$ and we can write it as $P=L^{\prime} / G^{\prime} . L$ is a finite covering group of $L^{\prime}$ (Lemma 2.2). As remarked at the end of $\$ 2, L$ and $G$ satisfy the conditions (P. 1-5). The subspace $\boldsymbol{P}=L_{-\beta}$ is diffeomorphic with $P$ by the exponential mapping restricted to $\boldsymbol{P}$, which is a $G$-equivariant diffeomorphism. Let $\gamma$ be the mapping of $G$ into $P$ defined by $\gamma(g)=\exp \circ \operatorname{ad}_{L}(g) z$, where $z$ is the one in (P. 5). $z$ is left fixed by $\operatorname{ad}_{L}(K)$. Thus $\gamma$ induces a mapping $\gamma^{*}$ of $M$ into $P$, which is an immersion by (1.2). Hence $\gamma^{*}(M)=G / K^{\prime}$, which is a $G$-orbit ( $\neq$ one point) in $P$, has $M=G / K$ as a finite covering homogeneous Riemannian manifold; in particular, it is locally symmetric. Putting $\Phi(G, K, L)=\left(\gamma^{*}(M), P\right)$, we thus have a mapping $\Phi$ of $\mathscr{A}$ into $\mathscr{P}$. It remains to show that $\Phi$ is surjective. Let $\left(M^{\prime}, P\right)=\left(G^{\prime} / K^{\prime}, L^{\prime} / G^{\prime}\right)$ be any element of $\mathscr{P}$. Let $p$ be a point of $M^{\prime}$ left fixed by the isotropy subgroup $K^{\prime}$. There exists a unique transvection $z$ in $\boldsymbol{L}=\boldsymbol{L}^{\prime}$ such that $p$ corresponds to $\exp (z)$ by the projection of $L^{\prime}$ onto $P ; z$ is a transvection in the sense that, $\boldsymbol{P}$ being symmetric by $(L, \beta), z$ belongs to $\boldsymbol{P}=\boldsymbol{L}_{-\beta}$. $\operatorname{ad}\left(K^{\prime}\right)$ operating on $\boldsymbol{L}$ leaves $z$ invariant. Thus $\boldsymbol{L}, \boldsymbol{G}\left(=\boldsymbol{G}^{\prime}\right)$ satisfy (P. 1-5). Let $H^{\prime}$ be the normalizer of $M_{-1}$ in $L^{\prime}$ (see Lemma 1.4). $H^{\prime}$ is a closed subgroup of $L^{\prime} . H^{\prime}$ contains $K^{\prime}$. $K^{\prime}$ is an open subgroup of $H^{\prime} \cap G^{\prime}$ which is compact. Thus $G^{\prime} / K^{\prime}$ is a finite covering space of $G^{\prime} / H^{\prime} \cap G^{\prime}$. On the other hand, the compact transformation group $G^{\prime}$ of $L^{\prime} / H^{\prime}$ has an open orbit. Hence, $L^{\prime} / H^{\prime}=G^{\prime} / H^{\prime} \cap G^{\prime}$. Therefore, some finite covering group $L^{\prime \prime}$ of $L^{\prime}$ operates on $G^{\prime} / K^{\prime}$ and contains $G^{\prime}$ naturally. Finally some finite covering homogeneous Riemann manifold $G / K$ of $G^{\prime} / K^{\prime}$ is a compact symmetric space on which some finite covering group $L$ of $L^{\prime \prime}$ operates containing $G . G / K$ and $L$ satisfy (A. 1,2). Since we have $L^{\prime} / G^{\prime}=L / G$ and $L^{\prime} / G^{\prime}$ is an irreducible symmetric space, they also satisfy (A. 3). (A. 4) is satisfied. Hence $(G, K, L)$ belongs to $\mathscr{A}$. Clearly we have $\Phi(G, K, L)=\left(M^{\prime}, P\right)$. Theorem 3.1 is proved.

LEMMA 3.2. Under the assumptions (A. 1-4), there exists an element $z$ in $\boldsymbol{P}$ such that (1) $z$ is ad(K)-invariant, (2) the vector field $z$ on $M$ vanishes at the origin $o, k(o)=o$, and $(3)\left(\operatorname{ad}_{L} z\right)^{2}$ is the identity operating on the tangent space $\boldsymbol{M}$ to $\boldsymbol{M}$ at $o$, where $\boldsymbol{P}$ denotes the orthogonal complement of $\boldsymbol{G}$ in $\boldsymbol{L}$ with respect to the Killing form of $L$.

Proof. $L$ and $G$ satisfy (P. 1-5) by Theorem 3.1. There exists an element $\boldsymbol{z}^{\prime}$ in $\boldsymbol{P}$ satisfying the conditions (1) and (3) in Lemma 3.2, by Lemma 1.3. Suppose $z^{\prime}$ does not vanish at $o$. Then $G$ has a one-dimensional center $G_{1}$ by (A. 3) and a property of symmetric spaces. Let $g_{1}$ be a nonzero element of $G_{1} \cdot \operatorname{ad}(K)$ leaves 
invariant and operates trivially on the subalgebra $L_{1}$ of $L$ generated by $\left\{z^{\prime}, g_{1}\right\}$. The orbit $L_{1}(o)$ is $G_{1}(o)$ which is a circle. Since $\left[z^{\prime}, g_{1}\right]$ does not vanish at $o$ by (3) satisfied by $z^{\prime}$, it follows that some nontrivial linear combination $z$ of $\left\{z^{\prime},\left[z^{\prime}, g_{1}\right]\right\}$ vanishes at $o$. This $z$ satisfies (1) and (2). $z$ also satisfies (3), if replaced by some scalar multiple, in view of the proof of Lemma 1.3.

Proposition 3.3. There exists a differentiable function $f$ on $M$ such that the one-form $d f$ equals the dual-form of the vector field $z$ in Lemma 3.2 with respect to some G-invariant Riemannian metric on $M$, under the assumptions (A. 1-4).

Proof. First we define the Riemannian metric. The space $\{\boldsymbol{n}(o) \mid \boldsymbol{n} \in[\boldsymbol{z}, \boldsymbol{M}]\}$, $\boldsymbol{M}=\boldsymbol{G}_{-\alpha}$, coincides with the tangent space $\{\boldsymbol{m}(o) \mid \boldsymbol{m} \in \boldsymbol{M}\}$ to $M$ at $o, K(o)=o$. The subspace $[z, M]$ of $L$ is left invariant by $\operatorname{ad}(K)$. Let $\pi$ be the orthogonal projection of $\boldsymbol{L}$ onto $[z, M]$ with respect to the Killing form $B$ of $\boldsymbol{L}$. We write $\langle\boldsymbol{x}(g(o)), \boldsymbol{y}(g(o))\rangle$ for $B\left(\pi \circ \operatorname{ad}(g-1)(x), \pi \circ \operatorname{ad}\left(g^{-1}\right)(y)\right)$ for any $x, y$ in $L$ and $g$ in $G$, where $\boldsymbol{x}(g(o))$ denotes the value taken by the vector field $\boldsymbol{x}$ on $M$ at the point $g(o)$. This gives rise to a well-defined field of quadratic forms ( $=$ a tensor field of covariant degree 2) on $M$; in fact, $B$ is invariant under $\operatorname{ad}(K)$, whose elements commute with $\pi$. Moreover, $\langle$,$\rangle defines a Riemannian metric on M$, since $B$ is positive definite on $[z, M]$ and $[z, M](o)$ is the tangent space to $M$ at $o$. This metric is invariant under $G$, as is readily seen. Now consider a function $f$ on $M$ defined by $f(g)(o)=B(\operatorname{ad}(g)(z), z)$ for all $g \in G$. We are proving $d f=z$, or, more precisely, $d f(\boldsymbol{x}(g(o)))=\langle\boldsymbol{x}(g(o)), z(g(o))\rangle$ for any $\boldsymbol{x}$ in $\boldsymbol{G}$ and $g$ in $G$. The left-hand side equals $x(g(o)) f=-B([x, \operatorname{ad}(g)(z)], z)=B\left(\left[z, \operatorname{ad}\left(g^{-1}\right)(x)\right], \operatorname{ad}\left(g^{-1}\right)(z)\right)$. Here we have $\left[z, \operatorname{ad}\left(g^{-1}\right)(x)\right]=\pi \circ \operatorname{ad}\left(g^{-1}\right)(x)$ due to the facts $\operatorname{ad}\left(g^{-1}\right)(x) \in \boldsymbol{G}$ $=\boldsymbol{K}+\boldsymbol{M}$ and $[\boldsymbol{z}, \boldsymbol{K}]=\{0\}$. Thus we obtain

$$
\begin{aligned}
x(g(o)) f & =B\left(\pi \circ \operatorname{ad}\left(g^{-1}\right)(x), \operatorname{ad}\left(\bar{g}^{1}\right)(z)\right) \\
& =B\left(\pi \circ \operatorname{ad}\left(g^{-1}\right)(x), \pi \circ \operatorname{ad}\left(g^{-1}\right)(z)\right)=\langle x(g(o)), z(g(o))\rangle,
\end{aligned}
$$

and Lemma 3.3 is proved.

LEMMA 3.4. Under the assumptions (A. 1-4), the involutive automorphism $\alpha$ of $G$ extends to that of $L$.

Proof. $\alpha$ induces an involutive isometry $\alpha^{\prime}$ of $M$. The function $f$ in Proposition 3.3 is invariant by $\alpha^{\prime} ; f \circ \alpha^{\prime}=f$ (by $\$ 16$ of Cartan [3]). Hence $z=d f$ is invariant by $\alpha^{\prime}$ (or, more precisely, the action of $\alpha^{\prime}$ on the vector fields). Thus $\alpha^{\prime}$ leaves invariant $L$ which is generated by $\{z\} \cup G$ in the Lie algebra of all vector fields on $M . \alpha^{\prime}$ is an automorphism of $L$. Since $L$ is connected and effective, the mapping $l \rightarrow \alpha^{\prime} l \alpha^{\prime}$ is thus an automorphism of $L$ which is an extension of $\alpha$.

Let $\mathscr{A}$ be as defined at the beginning of this section. Let $\mathscr{T}$ be the set of all pairs $(L, F)$ of a connected simple Lie group $L$ which is isomorphic with its ad- 
joint group and a closed subgroup $F$ of $L$ containing a line ( $=$ a one-dimensional vector group) in the center of $F$ such that $L / F$ is an affine symmetric space.

THEOREM 3.5. In the above notations, there exists a mapping $\Psi$ of $\mathscr{A}$ onto $\mathscr{T}$ such that, $\left(L^{\prime}, F^{\prime}\right)$ denoting $\Psi(G, K, L)$, (1) $L^{\prime}=\operatorname{ad}(L)$, and (2) $G^{\prime}=\operatorname{ad}(G)$ is a maximal compact subgroup of $L^{\prime}$ which is invariant under the involutive automorphism $\alpha^{\prime}$ of $L^{\prime}$, where ad is the projection of Lonto its adjoint group.

Proof. For any $(G, K, L)$ in $\mathscr{A}$, the involutive automorphism $\alpha$ of $G$ extends to that of $L$ which is also denoted by $\alpha$ (Lemma 3.4). Let $F$ be the set of all elements fixed under $\alpha$ belonging to $H$, where $H$ is the isotropy subgroup of $L$ at a point left fixed by $K, L / H=G / K$. The one-parameter subgroup $Z$ generated by $z$ in Lemma 3.2 is a line contained in $F$. From $H \cap G=K$, it follows that $F \cap G=K$. Since the center of $L$ is finite (Lemma 2.3), $G^{\prime}=\operatorname{ad}(G)$ is a maximal compact subgroup of $L^{\prime}=\operatorname{ad}(L)$ and $Z^{\prime}=\operatorname{ad}(Z)$ is a line. $\alpha$ induces an involutive automorphism $\alpha^{\prime}$ of $L^{\prime}$ and $F^{\prime}=\operatorname{ad}(F)$ is an open subgroup of the elements of $L$ which are invariant under $\alpha^{\prime}$. Since $F$ containing $Z$ is not compact, neither is $F^{\prime}$ (Lemma 2.3). $L / F^{\prime}$ is thus an affine symmetric space. Putting $\left(L^{\prime}, F^{\prime}\right)=\Psi(G, K, L)$, we thus obtain a mapping $\Psi$ of $\mathscr{A}$ into $\mathscr{T}$. Conversely, given $\left(L^{\prime}, F^{\prime}\right)$ in $\mathscr{T}$ we denote by $G^{\prime}$ a maximal compact subgroup of $L^{\prime}$ which is left invariant by the inner automorphism $\alpha^{\prime}$ of $L^{\prime}$ (Lemma 10.2 of Berger [2]). Put $K^{\prime}=F^{\prime} \cap G^{\prime}$. Then $M^{\prime}=G^{\prime} / K^{\prime}$ is a compact symmetric space. The line $Z^{\prime}$ in the center of $F^{\prime}$ is not contained in $K^{\prime}$; in fact, otherwise the closure of $Z^{\prime}$ would be a torus of dimension $>1$, which would be contained in the center of $F^{\prime}$, whence the compact form of $L^{\prime}$ would not be simple, and $G^{\prime}$ would therefore be simple so that the isotropy subgroup $K^{\prime}$ for the symmetric space $G^{\prime} / K^{\prime}$ would contain at most one one-dimensional subgroup in its center, a contradiction. It follows that, $\beta$ denoting the involutive automorphism of $L^{\prime}$ defining the symmetric Ispace $L^{\prime} / G^{\prime}$, there exists a line generated by some $z$ in $L_{-\beta}$ such that $K^{\prime}$ is contained in the centralizer of the line in $L^{\prime} . K^{\prime}$ is an open subgroup of the intersection $K^{\prime \prime}$ of that centralizer and $G^{\prime}$, since $G^{\prime}$ is a maximal compact subgroup of $L^{\prime}$. Theorem 3.1 thus applies; $\left(G^{\prime} / K^{\prime \prime}, L^{\prime} / G^{\prime}\right)$ belongs to $\mathscr{P}$ and there exists a $(G, K, L)$ in $\mathscr{A}$ with $\Phi(G, K, L)=\left(G^{\prime} / K^{\prime \prime}, L^{\prime} / G^{\prime}\right)$. Clearly we have $\Psi(G, K, L)=\left(L^{\prime}, F^{\prime}\right)$ and Theorem 3.5 is proved.

4. The structure theorems. We assume that $L$ and $G / K$ satisfy (A. 1-4) throughout this section. Then (P. 1-5) are satisfied with $\boldsymbol{K}=\boldsymbol{G}_{\alpha}$. Put $\boldsymbol{M}=\boldsymbol{G}_{-\alpha}$ and $\boldsymbol{P}=\boldsymbol{L}_{-\beta}$. We have seen that $\boldsymbol{L}$ is the direct sum $\boldsymbol{G}+\boldsymbol{P}=\boldsymbol{K}+\boldsymbol{M}+[\boldsymbol{z}, \boldsymbol{M}]+\boldsymbol{Q}$, where $\boldsymbol{Q}=[[\boldsymbol{z}, \boldsymbol{M}], \boldsymbol{M}]$ and $\boldsymbol{z}$ is as in Lemma 3.2. If $\boldsymbol{M}_{e}$ denotes $\{\boldsymbol{m}+e[\boldsymbol{z}, \boldsymbol{m}] \mid \boldsymbol{m} \in \boldsymbol{M}\}$, $e=+1$ or $-1, L$ is also the direct sum $M_{-1}+K+Q+M_{-1}$. By the adjoint representation of $L$ or $L$ on $L$, their elements operate on $L$. In this way the above subspaces are all $K$-modules. The elements of $\boldsymbol{L}$ are considered as vector fields on $M$. As before, we write, say, $M(o)$ for the space of the values taken by the 
vector fields in $\boldsymbol{M}$ at the point $o . M(o)$ is the tangent space $M_{o}$ to $M$ at $o$. Let $H$ be the isotropy subgroup $L$ leaving the point $o \in M, K(o)=o . z$ belongs to $\boldsymbol{H}$ and gives rise to a transformation of $M_{\boldsymbol{o}}$.

Lemma 4.1. Among the z's in Lemma 3.2 there exists a unique z such that the linear mapping $\nabla z(o)$ of the tangent space $M_{o}$ is the identity, and, in particular, that $\boldsymbol{M}_{+1}(o)$ is $M_{o}$, where $\boldsymbol{M}_{+1}=\{\boldsymbol{m}+[z, \boldsymbol{m}] \mid \boldsymbol{m} \in \boldsymbol{M}\}$ by this $z$.

Proof. Let $\boldsymbol{z}$ be as in Lemma 3.2. Then $\boldsymbol{F}=\boldsymbol{K}+\boldsymbol{Q}$ leaves $\boldsymbol{M}_{+1}$ and, as we show now, $\boldsymbol{H}$ invariant. (Note that $\boldsymbol{H}$ is the Lie algebra of $H$. We are going to show that $\boldsymbol{H}$ coincides with $\boldsymbol{M}_{-1}+\boldsymbol{F}$ in concurrence with the notation $\boldsymbol{H}$ in $\$ 1$.) For that, it is sufficient that $\boldsymbol{H}$ contains $\boldsymbol{F}$. Though this is obvious from $\alpha(z)=z$, this is seen from the fact that $[F, z]=\{0\}$ and the operation of $z$ on $M_{o}$ is nondegenerate ((3) of Lemma 3.2). $F$ is, moreover, irreducible on $\boldsymbol{M}_{+1}$ (Lemma 1.4). Thus $\boldsymbol{M}_{+1} \cap \boldsymbol{H}$ is either $\{0\}$ or $\boldsymbol{M}_{+1}$. In the latter case, we change the notation, writing $z$ for $-z$, which is obviously as in Lemma 3.2. So we may suppose that $\boldsymbol{M}_{+1} \cap \boldsymbol{H}=\{0\}$. Since $\boldsymbol{M}_{+1}$ has the same dimension as $\boldsymbol{M}$, it follows that $\boldsymbol{M}_{+1}(o)=M_{o}$ (and $\boldsymbol{L}$ is the direct sum $\left.\boldsymbol{H}+\boldsymbol{M}_{+1}\right)$. Any $z$ as in Lemma 3.2 is the identity transformation of $\boldsymbol{M}_{+1}$. To prove the uniqueness of $z$, let $\boldsymbol{z}^{\prime}$ be another one satisfying the conditions of the lemma. By $z(o)=0$ and by the direct sum decomposition $\boldsymbol{P}=[\boldsymbol{z}, \boldsymbol{M}]+\boldsymbol{Q}$, we see that $\boldsymbol{z}^{\prime}$ belongs to $\boldsymbol{Q}$. Therefore $\left[z, z^{\prime}\right]=0$ and $\operatorname{ad} z$ commutes with ad $z^{\prime}$. On the other hand, $\left[\boldsymbol{z}-\boldsymbol{z}^{\prime}, \boldsymbol{M}\right]$ is contained in $\boldsymbol{H}$, since both $z$ and $z^{\prime}$ are the identity transformation of $M_{\boldsymbol{o}}$. For any $\boldsymbol{m}$ in $\boldsymbol{M},\left[z-z^{\prime},[z-z, \boldsymbol{m}]\right]$ thus belongs to $\boldsymbol{H}$ and equals $\boldsymbol{m}-(\operatorname{ad} z)\left(\operatorname{ad} z^{\prime}\right)(\boldsymbol{m})$, which therefore belongs to $K$. Making $\operatorname{ad} z$ operate on this element of $\boldsymbol{K}$, we obtain $0=[z, m]-\left[z,\left[z^{\prime}, m\right]\right]=[z, m]-\left[z^{\prime},[z,[z, m]]\right]=[z, m]-\left[z^{\prime}, m\right]$. It follows that $\left[z-z^{\prime}, \boldsymbol{G} \cup\{z\}\right]=\{0\}$. Since $\boldsymbol{G} \cup\{z\}$ generates $\boldsymbol{L}$ and $\boldsymbol{L}$ is simple, $z-z^{\prime}$ belongs to the center of $L$, which is zero. Thus $z^{\prime}=z$.

TWO REMARKS ON THE METHOD OF THE PROOFS. The proofs hitherto given would be considerably shortened and made clear if one could generalize, to the case of 1-forms, the unique theorem of the zonal functions among spherical functions on a compact symmetric space. This problem is to show [5] that, for a symmetric space $M=G / K$, an irreducible representation of $G$, restricted to $K$, contains at most once the linear representation of $K$ on the tangent space $M_{o}$ to $M$ at $o, K(o)=o$. This problem is also equivalent to show that the space $\Omega$ of all 1-forms of $M$, on which $G$ operates naturally, contains no two different $G$-invariant subspaces which are isomorphic with each other as $G$-modules. Actually this is not true; in fact, if $M$ is an hermitian symmetric space, any $G$-submodule of $\Omega$ consisting of derived forms is isomorphic with some $G$-submodule of $\Omega$ consisting of coderived forms (the isomorphism being given by the almost complex structure). So some modification is necessary for the formulation of the problem. 
The arguments of space of functions in $\$ 2$ are not indispensable. They can be replaced by those of "proper" theory of Lie groups. Alternative proofs can be given in a more general way by using Mostow's theorem on maximal subgroups [On maximal subgroups of real Lie groups, Ann. of Math 74 (1961), 503-517] according to a forthcoming report of $\mathbf{H}$. Matsumoto (University of Tokyo).

THEOREM 4.2. If two groups $L$ and $L^{\prime}$ operate on $M=G / K$ and these all satisfy (A. 1-4) and if there exists an isomorphism of Lonto $L^{\prime}$ which leaves $G$ elementwise invariant, then $L, L^{\prime}$ are the same transformation groups of $M$.

Proof. Take the unique vector field $z$ [respectively, $z^{\prime}$ ] in $\boldsymbol{L}$ [respectively, $\boldsymbol{L}^{\prime}$ ] satisfying the condition of Lemma 4.1. Since $\boldsymbol{L}$ [respectively, $\boldsymbol{L}^{\prime}$ ] is generated by $\{z\} \cup \boldsymbol{G}$ [respectively, $\left\{z^{\prime}\right\} \cup \boldsymbol{G}$ ], we have to show that $z$ and $z^{\prime}$ coincide as vector fields on $M$. For any element $g$ in $G$, we have the obvious relation $z(g \cdot o)=d g\left(\operatorname{ad}\left(g^{-1}\right)(z)(o)\right)$, where $d g$ is the differential of the transformation $g$ of $M$ and $o$ is the point fixed by $K \cdot \operatorname{ad}\left(g^{-1}\right)(z)(o)$ lies in $L_{-\beta}$, the orthogonal complement of $\boldsymbol{G}$ in $\boldsymbol{L}$. Let $\pi$ be the (orthogonal) projection of $\boldsymbol{L}_{-\beta}$ onto $[\boldsymbol{z}, \boldsymbol{M}]$ with respect to the Killing form of $L$, or, equivalently, to the direct sum decomposition $\boldsymbol{L}_{-\beta}=[z, M]+[\boldsymbol{M},[z, M]]$, where $\boldsymbol{M}$ is the transvections of $M$ with respect to $o$. Since $Q=[M,[z, M]]$ leaves $o$ invariant, we obtain $\left.z(g \cdot o)=d g\left(\pi \circ \operatorname{ad}\left(g^{-1}\right) z\right)\right)(o)$. By using the fact (only) that $z$ is the identity transformation of the tangent space $M_{o}$ to $M$ at $o$, we can identify $[z, M]$ with $M_{o}$ which is identified with $\boldsymbol{M}$ in a way independent of $\boldsymbol{L}$ nor $\boldsymbol{L}^{\prime}$. So we have to show that $\phi\left(\pi \circ \operatorname{ad}\left(g^{-1}\right)(z)\right)=\pi^{\prime} \circ \operatorname{ad}\left(g^{-1}\right)\left(z^{\prime}\right)$ for any $g$ in $G$, where $\phi$ is the isomorphism: $\boldsymbol{L} \rightarrow \boldsymbol{L}^{\prime}$ given in the theorem. This equality follows from the fact that $L$ and $\boldsymbol{L}^{\prime}$ are isomorphic by $\phi$ and that $z$ and $z^{\prime}$ are determined from $\boldsymbol{L}, \boldsymbol{L}^{\prime}$ up to $( \pm 1)$-multiple in a way independent of the operations of $L, L^{\prime}$ on $M$ in view of the proof of Lemma 4.1, though $\phi$ in the equality must be replaced by $\phi \circ \beta$ in case $\phi(z)=-z^{\prime}$.

THEOREM 4.3. Under the assumptions (A. 1-4), we assume that the isotropy subgroup $H$ of $L$ at the point $o, K(o)=0$, is connected. Let $\rho$ be the (linear isotropy) representation of $H$ on the tangent space $M_{o}$ to $M$ at $o$. Then (1) $\rho(H)$ is irreducible, (2) there exists a closed subgroup $F$ of $H$ such that the restriction of $\rho$ to $F$ is an isomorphism of $F$ onto $\rho(H)$, (3) the kernel of $\rho$ is a vector group, $M_{-1}$, of dimension $n, n=\operatorname{dim} M$, (4) there exists an $n$-dimensional vector group, $M_{+1}$, of $L$, such that the orbit $M_{+1}(o)$ is homeomorphic with the $n$-dimensional euclidean space, (5) there exists an involutive automorphism $\beta$ of $L$ such that $\beta\left(M_{+1}\right)=M_{-1}, \beta(F)=F$, (6) $M_{+1} \cup M_{-1}$ generates $L,(7) R$ denoting the radical of $H, \rho(R)$ is the group of the real or "complex" scalar multiplication group, and (8) $K$ is a maximal compact subgroup of $F$.

(This theorem is based on Tanaka's conjecture.) 
Proof. Let $z$ be as in Lemma 4.1. Then $L, G$ and $z$ satisfy (P. 1-5). Let $\boldsymbol{M}, \boldsymbol{M}_{\boldsymbol{e}}$, respectively denote $\boldsymbol{G}_{-\alpha},\{\boldsymbol{m}+e[z, \boldsymbol{m}] \mid \boldsymbol{m} \in \boldsymbol{M}\}, e=+1$ or -1 . Then $\boldsymbol{M}_{-1}$ is contained in $\boldsymbol{H}$ and $\boldsymbol{M}_{+1}(o)=M_{o}$. From the latter we infer that the orbit at $o$ of the connected (abelian) subgroup $M_{+1}$ generated by $\boldsymbol{M}_{+1}$ is open in $M$. From the former, we have $d \rho\left(\boldsymbol{M}_{-1}\right)=\{0\}$ by the nullity of the covariant differentials of the elements (=transvections) of $\boldsymbol{M}$ at $o$. Hence the connected subgroup $M_{-1}$ generated by $\boldsymbol{M}_{-1}$ is contained in the kernel of $\rho$. Since $\boldsymbol{Q}=[\boldsymbol{M},[z, \boldsymbol{M}]]$ is contained in $\boldsymbol{H}$ (see the proof of Lemma 4.1), it follows from $\boldsymbol{M}_{+1}(o)=\boldsymbol{M}_{o}$ that $\boldsymbol{H}$ is the direct sum $\boldsymbol{M}_{-1}+\boldsymbol{Q}+\boldsymbol{K} . \boldsymbol{F}$ denoting $\boldsymbol{Q}+\boldsymbol{K}, d \rho(\boldsymbol{F})$ is equivalent to the restriction to $\boldsymbol{F}$ of the adjoint representation of $L$, operating on $\boldsymbol{M}_{+1}$, by $\boldsymbol{M}_{+1}(o)=M_{o}$ and (2) of Lemma 1.4. By this (2) again, $d \rho(\boldsymbol{F})$ is therefore irreducible and a fortiori $\rho(H)$ is irreducible and (1) of the theorem is proved. The connected group generated by $F$ in $L$ is a closed subgroup, since $F$ is the identity component of the centralizer of $\{z\}$. Any element $f$ of $F$ which is contained in the kernel of $\rho$ is contained in the centralizer of $\boldsymbol{M}_{+1}$ by the above. $f$ is also contained in the centralizer of $\boldsymbol{M}_{-1}$. Because $\boldsymbol{M}_{-1}$ is a dual space to $\boldsymbol{M}_{+1}$ by the canonical bilinear form $B\left(\boldsymbol{m}_{1}+\left[z, \boldsymbol{m}_{1}\right], \boldsymbol{m}_{2}-\left[z, \boldsymbol{m}_{2}\right]\right), \boldsymbol{m}_{1}, \boldsymbol{m}_{2} \in \boldsymbol{M}$ (which is negative definite as a bilinear form on $\boldsymbol{M}$ ), and $B$ is invariant under all automorphisms of $L$, and, in particular, the operations of $f(\operatorname{or} \operatorname{ad}(f))$ on $\boldsymbol{M}_{+1}$ and $\boldsymbol{M}_{-1}$ are adjoint with each other. Since $L / F$ is an affine symmetric space and the operation of $f$ on the tangent space to $L / F$ at the point fixed by is the above operation of $\operatorname{ad}(f)$ on $\boldsymbol{M}_{+1}+\boldsymbol{M}_{-1}$, we conclude that $f$ is contained in the center of $L$. Since $L$ is effective, this implies that $f$ is the identity element of $L$. Therefore $F$ is isomorphic with $\rho(F)$ by $\rho$. Since the connected group $H$ is $F M_{-1}$ (for $M_{-1}$ is a normal subgroup of $\boldsymbol{H}$ and $\boldsymbol{H}=\boldsymbol{F}+\boldsymbol{M}_{-1}$ ) and $\rho\left(M_{-1}\right.$ ) is the identity, we have $\rho(F)=\rho(H)$ and (2) is proved. The fact $H=F M_{-1}$ combined with the above gives that $M_{-1}$ is the kernel of $\rho$. Next, (5) is obvious if we take as $\beta$ the involutive automorphism of $L$ given by Lemma 2.5 ; in fact, $\beta$ is the identity on $M$ and we have $\beta(z)=-z$. Using (5), we prove that $M_{+1}$ and $M_{-1}$ are vector groups. If $M_{+1}$ (hence $M_{-1}$ ) is not a vector group, the abelian group $M_{+1}$ is a torus by the irreducibility of the automorphism $\operatorname{group} \operatorname{ad}(F)$ of $M_{+1}$ (see (1) and (2)). Since the orbit $M_{+1}(o)$ is open in $M, M$ must be a circle by (A. 3). Then $M_{-1}=\beta\left(M_{+1}\right)$ is a circle group, $F=\rho(F)$ is a line group, and so $L$ is a three-dimensional simple group containing a circle group $M_{-1}$ in the isotropy subgroup. By means of the exact sequence of the homotopy groups for the principal bundle $L \rightarrow L / H$, we are led to a contradiction. Thus $M_{+1}, M_{-1}$ are vector groups. Thus the proof of (3) is completed. The rest of the proof of (4) is analogous to the above; namely, if $M_{+1}(o)$ is not homeomorphic with a euclidean space, then it is a torus by (1) and (2) and again $M$ is a circle, whence the vector group $M_{+1}$ is not effective on $M$ contrary to (A. 2). (6) is obvious from arguments of Lie algebras: $\boldsymbol{M}_{+1}+\boldsymbol{M}_{-1}$ contains $\boldsymbol{M}$ and $[z, M]$, while $[\boldsymbol{M},[\boldsymbol{z}, \boldsymbol{M}]]$ is $\boldsymbol{Q}$ which contains $z$. Since $\rho(H)$ is irreducible, $\rho(H)$ is reductive 
and $\rho(R)$ is a group of scalar multiplications. Finally, (8) follows immediately from the observation that the elements of $d \rho(\boldsymbol{K})$ [respectively, $d \rho(\boldsymbol{Q})$ ] are skewsymmetric [respectively, symmetric] with respect to the Killing form of $L$ restricted to $\boldsymbol{M}_{+1}$.

THEOREM 4.4. Under the assumptions (A. 1-4), assume that the isotropy subgroup of $L$ is connected. Then $L$ operates transitively on the cotangent bundle $T^{*}(M)$ of $M$ in such a way that $T^{*}(M)$ becomes an affine symmetric space, $L$ is fibre-preserving and induces on the base manifold $M$ the transformation group $L$ in (A. 2). The restriction to $G$ of the operation of $L$ on $T^{*}(M)$ coincides with the operation of $G$ obtained from that of $G$ on $M$ by taking the codifferentials of the transformations of $M$ in $G$.

Proof. The notations being as before, $\boldsymbol{M}_{+1}$ is naturally identified with the tangent space $M_{o}$ to $M$ as an $F$-module. $M_{-1}$ is isomorphic with the dual space to $\boldsymbol{M}_{+1}$ as an $F$-module. Since $F$ contains $K, T^{*}(M)$ is thus isomorphic with the vector bundle $G \times{ }_{K} M_{-1}$ over $G / K=M$. This last bundle is isomorphic with the fibre bundle $L \times{ }_{H} H / F$ over $L / H=M$, whose bundle space is $L / F$. In fact, the principal bundle $G \rightarrow G / K$ is a subbundle of the principal bundle $L \rightarrow L / H$ and the restriction to $K$ of the operation of $H$ on $H / F$ coincides with that $K$ on $M_{-1}$, since the subgroup $M_{-1}$ of $L$ is a vector group (Theorem 4.3) and so the correspondence $\left(m_{-1}, f\right) \rightarrow m_{-1} f$ gives a diffeomorphism of $M_{-1} \times F$ onto $H$ and the exponential map of $\boldsymbol{M}_{-1}$ onto $M_{-1}$ is a $K$-equivariant diffeomorphism The rest of the theorem is now evident.

COROLlaRY. 4.5. Under the assumptions of (A. 1-4), the vector fields on $M$ contained in $L$ are uniquely determined by the values of them and of their first and second covariant derivatives taken at an arbitrary point.

The following theorem is obvious from the arguments up to here.

THEOREM 4.6. Under the assumptions (A. 1-4), there exists a differentiable function $f$ on $M$ such that (1) $f$ is an eigenfunction of the Laplacians (=G-invariant differentiable operators) of $M$, (2) $f$ is invariant under the reflection with respect to a point $o$ of $M$, in particular, the covariant differentiations of $f$ of any odd number of degrees vanish at o, (3) the second covariant differentiation $\nabla \nabla f$ coincides with the Riemannian metric tensor at ofor some G-invariant Riemannian metric of $M$, and (4) the Lie algebra of Lis generated by the transvections $M$ with respect to $o$ and the vector field which is dual to the exterior differentiation $d f$ with respect th the above Riemannian metric. $f$ is unique for a given $o$ (and the Riemannian metric).

5. The classification. The fundamental theorem enables us to classify all the pairs of $L$ and $M=[G / K$ with no distinction between $M$ and its covering manifolds in two ways. 
The first method is based on Theorem 3.5. and Berger's classification of locally affine symmetric spaces [2]. Take out from his list all the affine symmetric spaces $L / F$ such that $F$ contains a vector group in its center. That is all; $G$ and $K$ denoting a maximal compact subgroup of $L, F$ such that the involutive automorphism of $L$ leaves $G$ invariant, $L$ operates on $G / K$ satisfying (A. $1-4$ ) by virtue of Theorems 3.5 and 4.3 .

The second method is based on Theorem 3.1. Owing to E. Cartan, we know the linear isotropy representation of $G$ on the tangent space to the Riemannian symmetric space $L / G$. On the other hand, the representations of class 1 of $G$ with respect to any compact symmetric space $G / K$ are determined by an unpublished theorem of Sugiura (Osaka University). For $(L, M)$, the first said representation of $G$ is of class 1 , and vice versa. Now Sugiura's theorem reads as follows. Consider the Satake diagram (see [1]). For a white vertex $i$ next to a black, take the corresponding dominant form $\Lambda_{i}$. For a white vertex next to no black vertices take $2 \Lambda_{i}$. For a white vertex $i$ joined to another $j$ by a line with an arrow, take $\Lambda_{i}+\Lambda_{j}$. Then all these dominant forms (which are equal to the rank of $M$ in number) generate a semigroup which corresponds to the set of all irreducible representations of $G$ of class 1 .

Here is the list of all the pairs $(L, M)$, in which the same pair may appear under different articles. (See [2] for the notations of groups. Some groups have been replaced by locally isomorphic ones which are more popular.)

1. Group manifolds. $M=\mathrm{U}(n), \mathrm{SO}(n)$, and $\mathrm{Sp}(n) ; L=\mathrm{SU}^{n}(2 n), \mathrm{SO}^{n}(2 n)$ and $\operatorname{Sp}^{2 n}(n)$, respectively.

2. Products of spheres. $M=S^{p} \times S^{q}, p, q \geqq 0 . L=\mathrm{SO}^{p+1}(p+q+2)$.

3. Grassman manifolds. $M=\mathrm{U}(p+q) / \mathrm{U}(p) \times \mathrm{U}(q), \mathrm{SO}(p+q) / \mathrm{SO}(p) \times \mathrm{SO}(q)$, $\mathrm{Sp}(p+q) / \mathrm{Sp}(p) \times \mathrm{Sp}(q)$, and $\mathrm{Sp}(4) / \mathrm{Sp}(2) \times \mathrm{Sp}(2) . L=\operatorname{SL}(p+q, C), \operatorname{SL}(p+q, R)$, $\mathrm{SU}^{*}(2 p+2 q), E_{6}^{1}$.

4. Direct products of a circle and other symmetric spaces $M^{\prime}$. $M^{\prime}=\mathrm{SU}(2 n) / \mathrm{Sp}(n), \mathrm{SU}(n) / \mathrm{SO}(n), S^{q}$ and $E_{6} / F_{4}$. $L=\mathrm{SO}^{*}(4 n), \mathrm{Sp}(n, R), \mathrm{SO}^{2}(q+3)$ and $E_{7}^{3}$.

5. Hermitian symmetric spaces. $M=\mathrm{U}(p+q) / \mathrm{U}(p) \times \mathrm{U}(q), \mathrm{SO}(2+q) / \mathrm{SO}(2)$ $\times \mathrm{SO}(q), \mathrm{Sp}(n) / \mathrm{U}(n), \mathrm{SO}(2 n) / \mathrm{U}(n), E_{6} / \mathrm{SO}(2) \times \mathrm{SO}(10), E_{7} / \mathrm{SO}(2) \times E_{6} . L$ is the complexification of the isometry group.

6. The others. $M=F_{4} / B_{4}$, and $\mathrm{SU}(8) / \mathrm{Sp}(4) . L=E_{6}^{4}$ and $E_{7}^{1}$.

Using the classification, we obtain

THEOREM 5.1. Some finite covering space $G / K$ of a compact connected symmetric space $M_{1}$ is G-equivariantly and deformably immersed into another compact symmetric space $M_{2}=L_{u} / G$, if the diagram $M_{1} \rightarrow M_{2}$ is shown below. This is true instead of $M_{1}$ for the direct product of a circle and $M_{1}$ if $M_{1} \stackrel{T}{\rightarrow} M_{2}$ is shown below. ( $n, p$, and $q$ denote non-negative integers with $n=p+q$.) 
1. Unitary cycle. $\mathrm{U}(n) / \mathrm{U}(p) \times \mathrm{U}(q) \rightarrow \mathrm{SU}(n) \stackrel{T}{\rightarrow} \mathrm{U}(2 n) / \mathrm{U}(n) \times \mathrm{U}(n)$.

2. Mixed classical cycle. $\mathrm{SO}(2 n) / \mathrm{U}(n) \rightarrow \mathrm{SO}(2 n)$, $\mathrm{SO}(n) \rightarrow \mathrm{SO}(2 n) / \mathrm{SO}(n), \times \mathrm{SO}(n)$, $\mathrm{SO}(n) / \mathrm{SO}(p) \times \mathrm{SO}(q) \rightarrow \mathrm{SU}(n) / \mathrm{SO}(n) \stackrel{T}{\rightarrow} \mathrm{Sp}(n) / \mathrm{U}(n) \rightarrow \mathrm{Sp}(n)$

$\rightarrow \mathrm{Sp}(2 n) / \mathrm{Sp}(n) \times \mathrm{Sp}(n)$, $\mathrm{Sp}(n) / \mathrm{Sp}(p) \times \mathrm{Sp}(q) \rightarrow \mathrm{SU}(2 n) / \mathrm{Sp}(n) \stackrel{T}{\rightarrow} \mathrm{SO}(4 n) / \mathrm{U}(2 n)$.

3. Orthogonal series. $S^{p} \times S^{q} \rightarrow \mathrm{SO}(p+q+2) / \mathrm{SO}(p+1) \times \mathrm{SO}(q+1)$. $\mathrm{SO}(2+q) / \mathrm{SO}(2) \times \mathrm{SO}(q) \rightarrow \mathrm{SO}(2+q)$.

4. Freudenthal series. $F_{4} / \mathrm{SO}(9) \rightarrow E_{6} / F_{4} \stackrel{T}{\rightarrow} E_{7} / \mathrm{SO}(2) \times E_{6} \rightarrow E_{7}$.

5. The others. $\mathrm{Sp}(4) / \mathrm{Sp}(2) \times \mathrm{Sp}(2) \rightarrow E_{6} / \mathrm{Sp}(4)$.

$E_{6} / \mathrm{SO}(2) \times \mathrm{SO}(10) \rightarrow E_{6}$. $\mathrm{SU}(8) / \mathrm{Sp}(4) \rightarrow E_{7} / \mathrm{SU}(8)$.

6. Realizations of noncompact symmetric spaces as bounded domains. The following is well known for hermitian symmetric spaces.

THEOREM 6.1. If L operates on a compact symmetric space $G / K$ satisfying (A. 1-4), and if $G$ is semisimple, then the noncompact form $M^{\prime}=G^{\prime} / K^{\prime}$ of $M$ can be imbedded into $M$ so that $G^{\prime}$ is a subgroup of $L$ as a transformation group of $M$.

Proof. Let $\boldsymbol{G}=\boldsymbol{K}+\boldsymbol{M}$ be the Cartan decomposition. With $\boldsymbol{z}$ as in Proposition 3.2 , we put $\boldsymbol{G}^{\prime}=\boldsymbol{K}+[\boldsymbol{z}, \boldsymbol{M}]$. We have $[\boldsymbol{K}, \boldsymbol{z}]=\{0\}$ and $[[\boldsymbol{z}, \boldsymbol{x}],[\boldsymbol{z}, \boldsymbol{y}]]$ $=-[\boldsymbol{x}, \boldsymbol{y}] \in \boldsymbol{K}$ for any $\boldsymbol{x}, \boldsymbol{y}$ in $\boldsymbol{M}$. Thus $\boldsymbol{G}^{\prime}$ is a subalgebra of $\boldsymbol{L}$ and the above is the Cartan decomposition of the symmetric pair $\left(G^{\prime}, \alpha\right)$, where $\alpha$ is the extension to $\boldsymbol{L}$ of the involutive automorphism of $\boldsymbol{G}$ (Lemma 3.4). $\left(\boldsymbol{G}^{\prime}, \alpha\right)$ is dual to $(\boldsymbol{G}, \alpha)$, that is, its noncompact form. Let $G^{\prime}$ be the connected subgroup generated by $G^{\prime}$ in $L$, and let $K^{\prime}$ be $G^{\prime} \cap H$ where $H$ is the isotropy subgroup of $L$ at the point $o$ with $K(o)=o$. The Lie algebra of $K^{\prime}$ is $K . G^{\prime} / K^{\prime}=G^{\prime}(o)$ is open in $M$. $K_{o}^{\prime}$ denoting the identity component of $K^{\prime}, G^{\prime} / K_{o}^{\prime}$ is the noncompact form of $G / K$ and a covering manifold of $G^{\prime} / K^{\prime} . G^{\prime} / K^{\prime}$ becomes a homogeneous Riemannian manifold which is locally isometric with $G^{\prime} / K_{o}^{\prime}$ by the projection. Moreover, $G^{\prime} / K^{\prime}$ admits reflections (which coincide with those of $G / K$ restricted to $\left.G^{\prime} / K^{\prime}\right)$, since $G^{\prime}$ is invariant under $\alpha$. It follows that $G^{\prime} / K^{\prime}$ is the noncompact form of $M=G / K$. Besides, $G^{\prime}$ is effective on $G^{\prime} / K^{\prime}$, since $L$ is effective and $G^{\prime} / K^{\prime}$ is open. Theorem 6.1 is thus proved.

THeOREM 6.2(6). Suppose $L$ operates on $M=G / K$ satisfying (A. 1-4), where $G$ is assumed to be semisimple and $K$ connected. Then the noncompact form $M^{\prime}=G^{\prime} / K$ of $M$ is a bounded domain of $M_{+1}(o)$ by the identification $M^{\prime}=G^{\prime}(0)$.

(6) H. Matsumoto (University of Tokyo) also proved this, according to his letter. 
Proof. The method is so similar to that of Harish-Chandra described in $\$ 7$, Chapter VIII in [4] that we can omit the details. First, the existence of the inclusion $\exp [z, M] \subset M_{+1} F M_{-1}=M_{+1} F$, which means $G^{\prime}(o) \subset M_{+1}(o)$, is readily verified with the arguments on p. 318 of [4]. Thus there exists an imbedding $\zeta$ of $[z, M]$ into $\boldsymbol{M}_{+1}$ such that $\exp (\boldsymbol{m})$ belongs to $(\exp \zeta(\boldsymbol{m})) \cdot \boldsymbol{F M}$ for any $\boldsymbol{m} \in[z, M]$. On the other hand, the involutive automorphism $\alpha$ of $G$ extends to that of $L$ (Lemma 3.4), hence to the complexification of $L$. Denoting the extended one by the same $\alpha$, we see that $(\boldsymbol{K}+i[[z, \boldsymbol{M}], \boldsymbol{M}]+i \boldsymbol{M}+[\boldsymbol{z}, \boldsymbol{M}], \alpha)$ is a hermitian symmetric pair of noncompact type. The above $\zeta$ extends to an imbedding of $i \boldsymbol{M}+[\boldsymbol{z}, \boldsymbol{M}]$ into the complexification $\boldsymbol{M}_{+1}^{\boldsymbol{c}}$ of $\boldsymbol{M}_{+1}$, as shown with the same arguments. Now what Harish-Chandra finally showed is that the image of $\zeta$ is a bounded set in the euclidean space $\boldsymbol{M}_{+1}^{c}$. Therefore $\zeta([\boldsymbol{z}, \boldsymbol{M}])$ is a bounded subset in $\boldsymbol{M}_{+1}$, and the theorem is proved.

7. The critical manifolds of $f$. Throughout this section (A. 1-4) are assumed, and the notations in the preceding sections are freely used. Let $f$ denote the function mentioned in Theorem 4.6. It is explicitly given by $f(g(o))=B(\operatorname{ad}(g) z, z)$, $g \in G$ (see the proof of Lemma 3.3). Due to the equality $z=d f$, a point $m(o), \in \exp M$, is a critical point of $f$ if and only if $z$ vanishes at the point, or, equivalently, $m^{-1} z m \in \boldsymbol{H} \cap \boldsymbol{P}=\boldsymbol{Q}$. Since $m^{-1} z m$ is contained in $\boldsymbol{P}$, this last condition is characterized by the invariance of $m^{-1} z m$ under $\alpha$. Thus we have:

Lemma 7.1. A point $m(o), m \in \exp M$, of $M$ is a critical point of $f$ if and only if $\operatorname{ad}_{L}\left(m^{2}\right)(z)=z$.

Suppose $p$ is a critical point of $f$, i.e. $z$ vanishes at $p$. Since the vector field $z$ is invariant under $K$, any point on the $K$-orbit $K(p)$ is a critical point. On the other hand, for any vector field $v$ on $M, v(p)$ is annihilated by the Hessian of $f$ at $p$ if and only if $[v, z]$ vanishes at $p$. In particular, for any $\boldsymbol{m}$ in $\boldsymbol{M}$, this is equivalent to $[\boldsymbol{m}, z] \in \boldsymbol{H}_{p}$, where $\boldsymbol{H}_{p}$ is the Lie algebra of the isotropy subgroup of $L$ at $p$. Since $z$ belongs to $H_{p}$, this implies $m=[z,[z, m]] \in H_{p}$. Since $G$ is transitive on $M$, it follows that

LEMMA 7.2. A critical manifold is a K-orbit (or a connected component of it) which contains a critical point, and is always a nondegenerate critical manifold.

LEMMA 7.3. Every critical manifold is a symmetric space with respect to the induced metric from $M$.

Proof. To any point $p$ of $M$, the origin $o$ is carried by some $m \in \exp M$. Let $p$ be a critical point. The symmetric $\alpha_{p}$ of $M$ at $p$ is connected with the one $\alpha^{\prime}$ at $o$ by $\alpha_{p}=m \alpha^{\prime} m^{-1}=m^{2} \alpha^{\prime}$. Since $z$ is invariant under $\alpha^{\prime}$ (Theorem 4.6), it follows from Lemma 7.1 that $z$ is invariant under $\alpha_{p}$. On the other hand, the 
critical manifold $K(p)$ is given by the equation $z=0$ near $p$. Thus $K(p)$ is invariant under $\alpha_{p}$.

LeMma 7.4. The image of the immersion $\gamma^{*}$ given in Theorem 3.1 is naturally a symmetric space, or, what amounts to the same, Theorem 3.1 still holds when the family $\mathscr{P}$ is replaced by its subfamily consisting only of $(M, P)$ with $M$ (global) symmetric spaces.

We remember that $\gamma^{*}$ was defined by $\gamma^{*}(g(o))=g z g^{-1}=\exp \left(\operatorname{ad}_{L}(g) z\right), g \in G$, where $z$ is the point of $P$ corresponding to $\exp (z)$. Lemma 7.4 implies that $\gamma^{*}(M)$ satisfies (A. 1-4).

Proof of Lemma 7.4. Let $M^{\prime \prime}$ denote $\gamma^{*}(M)$. We define a reflection $\alpha^{\prime \prime}$ of $M^{\prime \prime}$ around $z$ by putting $\alpha^{\prime \prime}\left(g z g^{-1}\right)=\alpha(g) z \alpha(g)^{-1}$. We have to show that $\alpha^{\prime \prime}$ is well defined; i.e., $g z g^{-1}=z$ implies $\alpha(g) z \alpha(g)^{-1}=z$. Since $P=L / G$ is a symmetric space of noncompact type, $g z g^{-1}=z$ implies $\operatorname{ad}_{L}(g) z=z$. Naturally, $\operatorname{ad}_{L}(g)$ operates on the complexification $L^{c}$ of $L$. So $\operatorname{ad}_{L}(g)(i z)=i z, i^{2}=-1$. Thus $\operatorname{ad}_{L}(g)$ commutes with ad(iz) operating on $\boldsymbol{L}^{c}$. On the other hand, the isomorphism $\alpha$ of $G$ extends to that of $L$ (Lemma 3.4). Hence $\alpha$ induces an automorphism $\alpha_{L}$ of $\boldsymbol{L}$, which is nothing but $\exp (\pi \operatorname{ad}(i z))$ restricted to $\boldsymbol{L}$. It follows that $\operatorname{ad}_{L}(g)$ commutes with $\alpha_{L}$. Therefore we have $\alpha(g) z \alpha(g)^{-1}=\exp \left(\left(\operatorname{ad}_{L} \alpha(g)\right) z\right)$ $=\exp \left(\alpha_{L} \circ \operatorname{ad}_{L}(g) \circ \alpha_{L}^{-1}(z)\right)=\exp \left(\alpha_{L} \circ \alpha_{L}^{-1} \circ \operatorname{ad}_{L}(g)(z)\right)=\exp \left(\operatorname{ad}_{L}(g) z\right)=g z g^{-1}=z$. Thus $\alpha^{\prime \prime}$ is a well defined involutive diffeomorphism of $M^{\prime \prime}$. Now $M$ is a Riemannian covering space of $M^{\prime \prime} . \alpha^{\prime \prime}$ is clearly "covered" by the reflection $\alpha^{\prime}$ of $M$. Hence $\alpha^{\prime \prime}$ is an isometry with an isolated fixed point $z$. Lemma 7.4 is thus proved.

LEMma 7.5. If $M$ is imbedded in $P$ in the previously given way, i.e., $M=\gamma^{*}(M)$, then $o$ is the only point at which $f$ takes the greatest value. For any critical point $p$ and any geodesic $c$ joining $o$ to $p$, the extension of $c$ beyond $p$ by the same length is a closed geodesic, viz., $c$ is an arc of a circle in $M$ on which $o$ and $p$ are the antipodal points to each other.

Proof. Let $p$ and $c$ be as in the statement of the lemma. There exists a (unique) element $\boldsymbol{m}$ in $\boldsymbol{M}$ such that $c$ is an arc of the curve $(\exp t \boldsymbol{m})(o)$ with parameter $t$, corresponding to the interval $[0,1]$. (In our case the point $o=z$.) We put $c(t)=(\exp t m)(o)$. By Lemma 7.1 and the assumption $\gamma^{*}(M)=M$, we have $c(2)=o$ and the tangent $c^{\prime}(2)=c^{\prime}(0)=\boldsymbol{m}(o)$. If $A$ denotes $\operatorname{ad}_{L} \boldsymbol{m}$, we have $f(c(t))=B((\exp t A) z, z)$. We note that $(\exp t A) z$ is a curve in $\boldsymbol{P}$ on which $B$ is positive definite and that $\exp (t A)$ is orthogonal with respect to $B$. Since the expmap is injective on $\boldsymbol{P}$, it is now obvious that $f(c(t))$ or the inner product of $z$ with $(\exp t A) z$ (of equal length to $z$ ) with respect to $B$ takes the greatest value at $o$ and only at $o$. We have completed the proof of Lemma 7.5.

Let $T$ be a maximal connected flat totally geodesic submanifold of $M$ which contains the origin $o$. Then $T$ intersects with each critical manifold of $f$ in a finite number of points by Lemma 7.2. Lemma 7.5 thus implies there are at most 
$2^{r}$ critical manifolds where $r$ denotes the dimension of $T$ (which is the rank of $M)$. The number may be reduced by the action of the Weyl group of $M$ acting on $T$.

APPENDIX. In $\S 2$ our argument began with the assumptions (A. 1-4). But the study of the transformation group $L$ on a compact symmetric space $M$ is quite feasible under weaker ones, especially without the nongeometric condition (A.4) which requires $L$ to be simple, though nothing particularly interesting might be found. Here in the appendix we wish to offer a few remarks on this account. (We shall need for proofs what we have proved in preceding sections.) We will always assume (A. 1,2$)$ so that $L$ is a Lie transformation group of a compact symmetric space $M=G / K$ containing $G$ properly. It will be convenient for us first to observe an example.

ExAmple 1. Let $M^{\prime}$ and $M^{\prime \prime}$ be compact symmetric spaces and $M$ be the Riemannian product $T \times M^{\prime} \times M^{\prime \prime}$ where $T$ is a circle (=one-dimensional torus) group. Let $L^{\prime}, L^{\prime \prime}$ be semisimple Lie transformation groups on $M^{\prime}, M^{\prime \prime}$ containing the connected isometry groups $G^{\prime}, G^{\prime \prime}$, respectively. Assume $L^{\prime}=G^{\prime}$. The group $T \times L^{\prime} \times L^{\prime \prime}$ naturally operates on $M$. Let $\mathscr{F}$ be a finite-dimensional space of functions on $M^{\prime} . L^{\prime}$ naturally operates on $\mathscr{F}$. The elements $f$ of $\mathscr{F}$ can be considered as functions on $M$ via the projection of $M$ onto $M^{\prime}$. Let $t$ be a nonzero element of $\boldsymbol{T}$ considered as a vector field on $M$. The vector fields $f \boldsymbol{t}$ for all $f$ in $\mathscr{F}$ form a Lie algebra and generate a connected abelian transformation group of $M$. Now the connected Lie transformation group, $L$, generated by $R$ and $T \times L^{\prime} \times L^{\prime \prime}$ satisfies (A. 1,2) on $M$. Its radical is $R T$. (End of Example 1.)

Later we will prove that, under (A. 3), $L$ and $M$ are just as mentioned in Example 1 if $L$ is not semisimple and $M$ is replaced by some finite covering space. Moreover we will try to give some idea of the case where (A. 3) is not satisfied.

To discuss the case where $L$ is semisimple, we have to introduce a notion: as in the introduction, we say that a transitive Lie transformation group $L$ of a manifold $M$ is (locally) decomposable into $L^{\prime} \times L^{\prime \prime}$ if $L$ is the local direct product of two nondiscrete normal subgroups $L^{\prime}, L^{\prime \prime}$ whose orbits $L^{\prime}(x), L^{\prime \prime}(x)$ at every point $x$ of $M$ are closed and have the tangent spaces complementary with each other so that locally the transformation group $L$ is the direct product of the transformation group $L^{\prime}$ on $L^{\prime}(x)$ and $L^{\prime \prime}$ on $L^{\prime \prime}(x)$ and that $L$ is indecomposable if not.

When $L$ is decomposable into $L^{\prime} \times L^{\prime \prime}$ under $(\mathrm{A} .1,2)$, it is easy to see that $(A .1,2)$ are satisfied by $L^{\prime}$ and $L^{\prime \prime}$ acting on $L^{\prime}(x)$ and $L^{\prime \prime}(x)$ with metrics induced from $M$ except that at most one of $L^{\prime}, L^{\prime \prime}$ may coincide with the maximal connected isometry group.

Proposition 1. Assume (A.1,2) and that L is semisimple. Then L is simple if and only if $L$ is indecomposable. 
The "only if" part is obvious. Before the proof of the converse we make some preliminary observation, supposing that $L$ has a connected normal subgroup $N$. Then the $N$-orbits $N(x), x \in M$, form an $L$-invariant foliated structure, whose leaves $N(x)$ are the maximal integral manifolds of an $L$-invariant distribution $x \rightarrow N(x)$. (If, in particular, some $N(x)$ is open, then $N$ is transitive.) Since this distribution is invariant by $G$, it follows that the tangent spaces to the $N$-orbits are invariant under the holonomy group. Moreover, it is easily seen that every $N$-orbit has the tangent spaces parallel with each other along any curve on it, and thus the $N$-orbits are naturally symmetric spaces. If $N(x)$ is compact and different from $\{x\}$ and $M$, then it is also easy to see that $G$ is decomposed into $G^{\prime} \times G^{\prime \prime}$ in such a way that $G^{\prime}(y)=N(y)$ for any point $y$ of $M$. And $N(x)$ is compact if (A. 3) is satisfied or if the holonomy group leaves no nonzero tangent vectors to $N(x)$. When $N(x)$ is compact, we also note that the orbit space $M / N$ is a (global) symmetric space in a natural fashion. And $L$ becomes a (not necessarily effective) transformation group of $M / N$.

We feel it worth noting that if $L$ is primitive on $M$ (i.e., $L$ leaves invariant no nontrivial foliated structure on $M$ ) then (A. 4) is satisfied, and so is (A. 3). In fact, otherwise, $L$ would have a proper nondiscrete normal subgroup whose orbits would form an $L$-invariant foliated structure.

Now to prove Proposition 1, we assume that $L$ is not simple. We will first show that

(1) Any connected simple normal subgroup $L^{\prime}$ of $L$ is not transitive on $M$. Proof of (1). Suppose $L^{\prime}$ is transitive. Let $L^{\prime \prime}$ be the connected normal subgroup of $L$ such that $L$ is the local direct product of $L^{\prime}$ and $L^{\prime \prime}$. Since the center of $L$ is finite (Lemma 2.2 and the remark after its proof), we have the local product decomposition $G=G^{\prime} G^{\prime \prime}$, where $G^{\prime}, G^{\prime \prime}$ are the intersections of $G$ with $L^{\prime}, L^{\prime \prime}$ respectively. Neither $G^{\prime}$ nor $G^{\prime \prime}$ is discrete. We consider the case (i): $L^{\prime} \neq G^{\prime}$. Since $G^{\prime \prime}$ is a compact normal subgroup of $L^{\prime} G^{\prime \prime}, L^{\prime}$ is a transformation group of the orbit space $M / G^{\prime \prime}$, denoted by $M^{\prime}$. Since $L^{\prime}$ is simple, $L^{\prime}$ is almost effective on $M^{\prime}$ unless $G^{\prime \prime}$ is transitive (and hence $L=G$ ). To save notations, we assume that $L^{\prime}$ is effective on $M^{\prime}$. $L^{\prime}$ on $M^{\prime}$ satisfies (A. 1-4). On the other hand, the isotropy subgroup $N^{\prime}$ of $L^{\prime}$ on $M$ at $x$, a point of $M$, is a subgroup of the isotropy subgroup $H^{\prime}$ of $L^{\prime}$ on $M^{\prime}$ at the point $G^{\prime \prime}(x) . H^{\prime}$ is transitive on $G^{\prime \prime}(x)$ as a transformation group of $M$, because $L^{\prime}$ is transitive on $M$. Since the elements of $H^{\prime}$ commute with those of $G^{\prime \prime}$, both $H^{\prime}$ and $G^{\prime \prime}$ are simply transitive on $G^{\prime \prime}(x)$. Hence $N^{\prime}$ is a normal subgroup of $H^{\prime}$ and $H^{\prime} / N^{\prime}$ is isomorphic with $G^{\prime \prime}$. By Theorem 4.3 (or its proof), $H^{\prime}$ must contain a subgroup which is isomorphic with $G^{\prime \prime}$ and which, moreover, is not contained in $N^{\prime}$ but in (the isotropy subgroup at $G^{\prime \prime}(x)$ of) $G^{\prime}$ operating on $M^{\prime}$. Therefore $G^{\prime}$ is transitive on $G^{\prime} G^{\prime \prime}(x)=G(x)=M$. It follows that the symmetric space $M$ is a local Riemannian product of the group manifold $G^{\prime \prime}$ and some symmetric submanifold. Thus, if $G^{\prime \prime}$ is a toral group, $G^{\prime}$ having a discrete intersection with $G^{\prime \prime}$ cannot be transitive 
on $M$. If $G^{\prime \prime}$ is not a toral group, $G^{\prime}$ contains a normal and nondiscrete subgroup whose orbits are contained in those of $G^{\prime \prime}$. Hence that subgroup acts trivially on $M^{\prime}$, which is also a contradiction. And the case (i) never occurs. Now we consider the other case (ii): $L^{\prime}=G^{\prime} . M$ is then a simple group manifold, since $G^{\prime}$ is now simple and $G^{\prime \prime}$ is not discrete. In particular, $G^{\prime \prime}$ is transitive. Since the elements of $L^{\prime \prime}$ commute with those of $G^{\prime}$ which is transitive, they must belong to $G^{\prime \prime}$. Thus we have $L=G$, a contradiction. (1) is proved.

Proof of Proposition 1. We have been assuming that $L$ is not simple. Given arbitrarily a connected normal simple subgroup of $L$, we denote by $L^{\prime}$ the maximal connected subgroup of $L$ which has the same orbits as the given one. $L^{\prime}$ is a normal subgroup of $L$, which is a proper subgroup by (1). $L$ is a local direct product of $L^{\prime}$ and some normal subgroup, $L^{\prime \prime}$, of $L$. The transitive group $G$ is decomposable into $G^{\prime} \times G^{\prime \prime}$ where these subgroups are the intersections of $G$ with $L^{\prime}, L^{\prime \prime}$, since $L$ has the center finite and $G^{\prime}$ must be transitive on $L^{\prime}$-orbits by the definition of $L^{\prime}$. In order to prove that $L$ is decomposed into $L^{\prime} \times L^{\prime \prime}$ it suffices to show that the triviality of the intersection $I$ of the tangent spaces $L^{\prime}(x)$ and $L^{\prime \prime}(x)$ to $L^{\prime}(x), L^{\prime \prime}(x)$ at a point $x$ of $M$. Clearly there exists a subspace $C$ of the center of $\boldsymbol{G}^{\prime}$ such that $\boldsymbol{I}=\{\boldsymbol{c}(x) \mid \boldsymbol{c} \in \boldsymbol{C}\}$ (since $G^{\prime}$ is the maximal connected isometry group of the symmetric space $G^{\prime}(x)=L^{\prime}(x)$ and the isotropy subgroup of $G^{\prime}$ is trivial on $I$ ). For each vector field $c$ in $C$, there exists a vector field $v$ in $L^{\prime \prime}$ such that $v(x)=c(x)$. Both $v$ and $c$ are invariant by $G^{\prime}$. Hence we have $v=c$ on $L^{\prime}(x)$. This implies $\left[\boldsymbol{L}^{\prime}, \boldsymbol{c}\right]=0$ on $L^{\prime}(x)$, or, in other words, $C$ is an abelian ideal in the semisimple Lie algebra $L^{\prime}$. Thus $\boldsymbol{C}=0$, whence $\boldsymbol{I}=0$, and the proposition is proved.

Proposition 2. Assume (A. 3) beside (A. 1,2). If $L$ is not semisimple, then the identity component $T$ of the center of $G$ is not trivial and contained in the center of $L$. Moreover, $S$ denoting a maximal connected semisimple subgroup of $L, S T$ is transitive and decomposable into $S \times T$. The radical $R$ of $L$ is given by an invariant space of functions on $M / T$ as in Example 1.

Proof. The $R$-orbits are circles by (A. 3 ). since the $R$-orbits are compact symmetric spaces by (A. 3) which, as solvmanifolds, have euclidean spaces as the universal covering spaces. In particular, $T$ is not trivial but a circle group. Let $R^{\prime}$ be the maximal connected subgroup of $L$ which has the same orbits as $R$. $R^{\prime}$ is a normal subgroup of $L$ containing $R T$. As a transformation group of its orbit, $R$ is a circle group (see (2) below), which is a normal subgroup of a connected Lie transformation group $R^{\prime}$ of the orbit. This implies that as such $R$ is contained in the center of $R^{\prime}$. Still as such $R^{\prime}$ is thus an abelian group. Hence $R^{\prime}$ is abelian. Thus the normal subgroup $R^{\prime}$ coincides with $R$. Hence $R$ contains $T$ in particular. Thus $[T, L] \subset[R, L] \subset R$. Since $R$ is abelian, it follows $[T,[T, L]]=0$. Next we prove $[\boldsymbol{L}, T]=0$. Any element $\boldsymbol{l}$ of $L$ is of the form $f \boldsymbol{t}+\boldsymbol{u}$ where $f$ is a function on $M, t$ is a fixed nonzero element of $\boldsymbol{T}$ and $\boldsymbol{u}$ is a 
vector field on $M$ which is perpendicular to the $R$-orbits. Locally $\boldsymbol{u}$ is a linear combination (with functions as coefficients) of vector fields in the semisimple part of $G$. Hence $[t, u]=0$ since $[t, l]$ belongs to $R$. By $[T[T, L]]=0$ proved above, $f$ satisfies $\boldsymbol{t} t f=0$. A function on a circle with this property is necessarily a constant. Thus we obtain $[t, l]=0$. In particular, $S T$ is a local direct product of $S$ and $T$. We can assume without loss of generality that $S$ contains the semisimple part $G^{\prime}$ of $G$. To prove the contrary, we assume the orbits of $S$ are different from those of $G^{\prime}$. Consider the operation of $S$ on $M / T$. It satisfies (A. 1,2). By Proposition 1 and the argument in its proof, $G^{\prime}$ must be transitive on $M$, a contradiction. Therefore $S T$ is decomposable into $S \times T$. Now any element $\boldsymbol{r}$ of $\boldsymbol{R}$ is of the form $f \boldsymbol{t} . f$ is constant along each $T$-orbit by $[R, T] \subset[\boldsymbol{L}, \boldsymbol{T}]=0$. Hence $f$ can be considered as a function on $M / T$. The space of all these functions $f$ are invariant under $L$; in fact, $(l f) t=[l, f t] \in R$ for every $l$ in $L$.

(2) In general, a connected solvable Lie transformation group $R$ of a circle is a circle group if it is effective.

This fact is more or less well known, but a proof is given here, with the induction on the dimension of $R$. The commutator subgroup $C$ has the dimension strictly smaller than that of $R$. When $C$ is discrete, $R$ is abelian and certainly a circle group, for otherwise $R$ would not be effective. Since $C$ is a normal subgroup and $R$ is effective, $C$ must be transitive on the circle. By the assumption of the induction $C$ is thus a circle group. It follows that $C$ is contained in the center of $R$, as is easily see from the completely reducible $\operatorname{ad}_{R} C$. Hence the isotropy subgroup of $R$ centralizes the transitive group in particular, and therefore it must reduce to the identity.

To understand better the meaning of Example 1, we note:

(3) Under the assumptions (A. 1-4), a finite-dimensional space $\mathscr{F}$ of functions on $M$ consists of constants only if it is invariant by $L$.

Proof. Let $z$ be the vector field on $M$ mentioned in Lemma 4.1. Consider any integral curve $c$ of $z$ passing through a point near $o$ but not $o . c$ is contained in an integral curve of some vector field $\boldsymbol{v}$ in $\boldsymbol{M}_{+1}$. On this curve any function $f$ in $\mathscr{F}$ is bounded. $f$ converges when a point on $c$ tends to $o$ and simultaneously satisfies two linear homogeneous differential equations with constant coefficient with respect to (different) coordinate systems on $c$; in fact, since $\mathscr{F}$ is finite dimensional, there exist polynomials $P$ and $Q$ for each $f$ such that $P(z) f=0$ and $Q(v) f=0$, where, by definition, $z f$ and $v f$ are the functions obtained by the usual actions of vector fields on functions and $z^{k+1} f=z\left(z^{k} f\right)$, etc. Thus $f$ must be constant.

Finally, we will give an example in which $M$ is a torus and $L$ is a solvable group. A torus $M$ is a quotient space $V / D$ of a vector group $V$ over a lattice group $D$. Given a linear form $\alpha \in V^{*}$ on the Lie algebra $V, c_{\alpha}$ and $s_{\alpha}$ shall denote the functions on $V$ defined by $c_{\alpha}(\exp \boldsymbol{x})=\cos 2 \pi \cdot \alpha(\boldsymbol{x})$ and $s_{\alpha}(\exp \boldsymbol{x})=\sin 2 \pi \cdot \alpha(\boldsymbol{x})$, respectively. 
EXAMPLE 2. Let $\Sigma$ be a finite subset of $V^{*} \times V$ such that $\alpha(D)$ consists of integers for each $(\alpha, x) \in \Sigma$ and that $\alpha(y)=0$ for any two $(\alpha, x),(\beta, y)$ (distinct or not) in $\Sigma$ (hence $\alpha(\boldsymbol{x})=\beta(\boldsymbol{x})=\beta(\boldsymbol{y})=0$. Let $\boldsymbol{L}$ be the space spanned by $\boldsymbol{V}$ and the vector fields $c_{\alpha} x, s_{\alpha} x$ for all $(\alpha, x)$ in $\Sigma . L$ is then a solvable Lie algebra. Each vector field in $\boldsymbol{L}$ is invariant by the natural action of $D$. Thus $\boldsymbol{L}$ generates on the torus $M=V / D$ a connected solvable Lie transformation group satisfying (A. 1,2).

It would not be hard to show that, conversely, a connected solvable Lie transformation group $L$ on a torus $M$ subject to $($ A. 1,2$)$ is obtained in this way.

\section{BIBLIOGRA PHY}

1. S. Araki, On root systems and an infinitesimal classification of irreducible symmetric spaces, J. Math. Osaka City Univ. 13 (1962), 1-34.

2. M. Berger, Les espaces symmétriques non compacts, Ann. Sci. École Norm. Sup. 74 (1957), 85-177.

3. E. Cartan, Sur la détermination d'un système orthogonal complet..., Rend. Circ. for Arc. 53 (1929), 217-252.

4. S. Helgason, Differential geometry and symmetric spaces, Academic Press, New York, 1961.

5. T. Nagano, On the minimum eigenvalues of the Laplacians in Riemannian manifolds Sci. Papers College Gen. Ed. Univ. Tokyo 11 (1961), 177-182.

6. - On fibered Riemann manifolds, Sci. Papers College Gen. Ed. Univ. Tokyo 10 (1960), 17-27.

7. - The conformal transformation on a space with parallel Ricci tensor, J. Math. Soc. Japan 11 (1959), 10-14.

8. - The projective transformation on a space with parallel Ricci tensor, Ködai Math. Sem. Rep. 11 (1959), 131-139.

UNIVERSITY OF CALIFORNIA,

Berkeley, California

UNIVERSITY OF TOKYO,

TOKYO, JAPAN 